・森林动态监测样地专题・

\title{
宝天曼落叶阔叶林土壤细菌多样性
}

\author{
赵爱花 ${ }^{1,2} \quad$ 杜晓军 ${ }^{1 *}$ 藏 $\quad$ 婧1 张守仁 $^{1} \quad$ 焦志华 $^{3}$ \\ 1 (中国科学院植物研究所植被与环境变化国家重点实验室, 北京 100093) \\ 2 (中国科学院大学, 北京 100049) \\ 3 (中国矿业大学(北京)化学与环境工程学院, 北京 100083)
}

\begin{abstract}
摘要: 土壤微生物在森林生态系统中起着重要作用。高通量测序方法的出现为进一步认识土壤微生物提供了契 机。本文利用Illumina Miseq高通量测序技术对宝天曼森林土壤的细菌多样性进行了初步研究。结果显示: 在31个 采样点内, 随着采样点增加, 检测出不同分类水平的土壤细菌类群也在增多, 当采样点达到 31 个时, 检测出的土 壤细菌类群达到45门163纲319目495科785属和42,632个OTU; 31个土壤样品中所检测出的细菌类群平均有34.2门 114.7纲215.2目323.7科446.6属5,924.7个OTU, 其中门、纲、目分类水平上的优势类群(所占比例)分别为变形菌门 (Proteobacteria)(38.30\%)、 $\alpha$-变形菌纲( $\alpha$-Proteobacteria)(18.08\%)、根瘤菌目(Rhizobiales)(10.62\%)。这些初步研究结 果表明在一定程度上宝天曼森林土壤有较高的细菌多样性水平, 为进一步认识森林土壤细菌多样性与植物多样性 关系等奠定了基础。
\end{abstract}

关键词: 森林, 土壤微生物, 细菌多样性, 高通量测序, 变形菌门, $\alpha$-变形菌纲, 根瘤菌目, 宝天曼国家级自然保护区

\section{Soil bacterial diversity in the Baotianman deciduous broad-leaved forest}

\author{
Aihua Zhao ${ }^{1,2}$, Xiaojun $\mathrm{Du}^{1 *}$, Jing Zang ${ }^{1}$, Shouren Zhang ${ }^{1}$, Zhihua Jiao ${ }^{3}$ \\ 1 State Key Laboratory of Vegetation and Environmental Change, Institute of Botany, Chinese Academy of Sciences, Bei- \\ jing 100093 \\ 2 University of Chinese Academy of Sciences, Beijing 100049 \\ 3 School of Chemical and Environmental Engineering, China University of Mining \& Technology (Beijing), Beijing \\ 100083
}

\begin{abstract}
Soil microbes play an essential role in forest ecosystems. The development of the high-throughput sequencing method provides an invaluable opportunity to further understand soil microbial communities. In this study, we investigated soil bacterial diversity in the Baotianman forest using the Illumina Miseq platform. Results showed that in the 31 soil sampling points, the number of bacterial taxa detected at different classification levels increased with increasing number of sampling points. When all 31 samples were considered, the number of relative bacterial groups included 45 phyla, 163 classes, 319 orders, 495 families, 785 genera and 42,632 OTUs. The mean values of relative bacterial taxa in the 31 samples were 34.2 phyla, 114.7 classes, 215.2 orders, 323.7 families, 446.6 genera, and 5,924.7 OTUs. At the classification level of phylum, class and order, the dominant groups were Proteobacteria (38.30\%), $\alpha$-Proteobacteria (18.08\%), and Rhizobiales (10.62\%), respectively. These preliminary findings suggest that Baotianman forest soils have high levels of bacterial diversity to some degree and provide basic information and knowledge to further understand the relationship between soil bacterial diversity and plant diversity and other related scientific questions.
\end{abstract}

Key words: forest, soil microbe, bacterial diversity, high-throughput sequencing, Proteobacteria, $\alpha$-Proteobacteria, Rhizobiales, Baotianman National Nature Reserve

微生物是地球上多样性最高的生命形式(Fierer \& Jackson, 2006), 其分布也最为广泛, 几乎分布到
地球上的所有生境(郭良栋, 2012)。土壤是最丰富的 微生物资源库, 其微生物数量庞大、生态功能活跃

收稿日期: 2015-02-10 接受日期: 2015-05-22

基金项目: 国家自然科学基金(31270642, 31370586)

* 通讯作者 Author for correspondence. E-mail: xjdu@ibcas.ac.cn 
(蔡晨秋等, 2011)。土壤微生物在生态系统中扮演着 重要的角色, 调节植物健康和土壤肥力, 影响许多 重要的生态过程, 比如植物对养分的获取, 氮、碳、 磷、硫、金属元素等物质的生物地球化学循环, 土 壤的形成, 环境污染物的生物降解等(van der Heijden et al., 2008; He et al., 2011; Fierer et al., 2012)。 而细菌又在土壤微生物中占绝对优势(顾峰雪等, 2000; 赵先丽等, 2007; Delmont et al., 2012; Fierer et al., 2012), 其所占比例与土壤理化性质、土壤深 度等诸多因素有关, 有时甚至高达 $95 \%$ 以上(顾峰雪 等, 2000)。土壤细菌又是陆地生态系统中几乎所有 生物地球化学循环的重要驱动者, 参与了土壤中大 多数养分的转化(Nacke et al., 2011)。相对于农田和 草地生态系统来说, 森林生态系统中的土壤细菌群 落仍然没有被充分研究(Li et al., 2014)。森林生态系 统地下具有丰富的微生物资源: 在门的分类水平 上, 森林土壤细菌多样性虽不如草地(Nacke et al., 2011), 但却高于农田(Roesch et al., 2007)。有研究报 道, 不同的自然森林类型其土壤细菌的优势类群不 同(Li et al., 2014), 有研究发现变形菌是温带森林 土壤细菌中含量最丰富的类群(Zhang et al., 2014b), 也有研究发现酸杆菌是温带森林土壤细菌中相对 丰度最高的类群(Fierer et al., 2012)。

以往对于土壤微生物的研究方法有很多, 如传 统的平板培养法、Biolog微平板法、磷脂脂肪酸谱 图分析法(PLFA)、基于核酸分子杂交技术的方法、 基于PCR技术的方法(蔡晨秋等, 2011)及Sanger测序 等。这些方法的应用促进了微生物生态学等相关学 科的发展, 但是这些方法仅能测定土壤中部分微生 物种类, 很难发现稀有种类微生物, 因此土壤微生 物仍常常被看作“黑箱”(Tiedje et al., 1999; Macrae, 2000; Fernando \& Li, 2012)。高通量测序技术(也被称 为第二代测序技术)的发展, 为人们认识土壤微生 物多样性提供了新的契机。在多种高通量测序技术 中, 罗氏454测序技术和Illumina测序技术的应用比 较多, 而Illumina测序技术具有低错误率、低成本和 高通量的优点(孙欣等, 2013), 使得简单、快速、较 准确地获取土壤微生物信息成为可能, 现已基本取 代454测序技术。本研究使用Illumina测序技术开展 了森林土壤微生物多样性研究。

森林土壤细菌或微生物群落调查的第一步是
获得一定数量的森林土壤样品, 且这些样品能够代 表它们所在的群落。目前的研究中有每样地或样方 采集1个(Fierer \& Jackson, 2006; Fierer et al., 2012; Zhang et al., 2014a, b)、3个(Jiang et al., 2010; Wu et al., 2013)、10个(Hackl et al., 2004, 2005)、36个(Li et al., 2014)等不同数量的土壤样品。那么究竟多少个 土壤样品才能充分反映森林土壤微生物群落的信 息呢? 这是一个基本且重要的问题(Wooley et al., 2010), 同时也是一个需要进一步研究的问题。

宝天曼国家级自然保护区地处暖温带向北亚 热带的过渡区, 主要植被类型为暖温带落叶阔叶 林, 地理位置特殊、生境类型多样、生物多样性十 分丰富(宋朝枢,1994), 是进行森林生态学和生物多 样性相关研究的理想场所。本文采用Illumina公司 Miseq测序仪对位于宝天曼保护区内的25 ha森林固 定样地土壤细菌多样性进行了初步研究, 以期回答 以下问题: (1)宝天曼森林土壤细菌多样性水平如 何？(2)宝天曼森林土壤细菌的门、纲、目、科、属 等不同分类水平的优势类群有哪些? 各类群相对 丰度是多少? (3)取样数量对土壤细菌多样性检测 结果有何影响? 即随着取样数量增多, 土壤细菌多 样性如何变化? 对这些问题的回答将有助于土壤 细菌多样性及其与植物多样性的关系等的进一步 研究。

\section{1 材料与方法}

\section{1 研究区概况与土壤样品采集}

宝天曼国家级自然保护区位于河南省南阳市 内乡县境内, 地理坐标为 $33^{\circ} 25^{\prime}-33^{\circ} 33^{\prime} \mathrm{N}, 111^{\circ} 53^{\prime}-$ $112^{\circ} \mathrm{E}$ 之间。地处南暖温带向北亚热带的过渡区域, 是中原地带少有的保存较完整的森林, 区域内生境 类型多样、物种丰富。年均气温 $15.1^{\circ} \mathrm{C}$, 年均降水量 $885.6 \mathrm{~mm}$, 年均相对湿度68\%。区内土壤主要分为山 地棕壤土、山地黄棕壤土和山地褐土 3 个类型, 土壤 pH值5.5-7.2 (宋朝枢, 1994)。25 ha (500 m × $500 \mathrm{~m})$ 宝天曼森林动态监测样地建于2009年, 位于宝天曼 国家级自然保护区内, 主要森林类型为暖温带落叶 阔叶林。我们于2013年夏季在宝天曼样地中选取了 31 个采样点(图1), 在每个点附近的土壤表层(深度 为 $0-10 \mathrm{~cm}$ ) 用土钻取 10-15钻土, 混合均匀、去除根 和石头, $-80^{\circ} \mathrm{C}$ 保存用于 $\mathrm{DNA}$ 的提取和后续实验。 


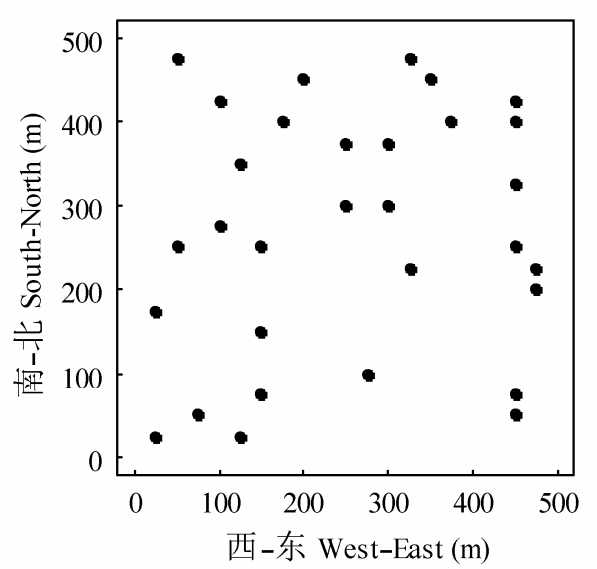

图1 25 ha宝天曼森林动态监测样地31个土壤微生物采样 点位置

Fig. 1 Location of the 31 soil samples in the 25 ha Baotianman Forest Dynamics Plot

\subsection{DNA提取、扩增和高通量测序}

土壤微生物宏基因组提取用的是 PowerSoil ${ }^{\circledR}$ DNA Isolation Kit (美国MoBio公司)试剂盒。采用引 物 515F (5'-GTGCCAGCMGCCGCGGTAA-3') 和 806R (5'-GGACTACHVGGGTWTCTAAT-3')对16S rRNA基因的V4高变区进行PCR扩增, 所用的方法 为样品标记法(Caporaso et al., 2011, 2012)。PCR反 应体系50 $\mu \mathrm{L}$ : $0.5 \mu \mathrm{L}$ TaKaRa LA Taq (5 U/ $\mu \mathrm{L}), 5 \mu \mathrm{L}$ 10×LA PCR Buffer II ( $\mathrm{Mg}^{2+}$ Plus), $5 \mu \mathrm{L}$ dNTP Mixture (各 $2.5 \mathrm{mM}), 2 \mu \mathrm{L}$ 模板DNA, $1 \mu \mathrm{L}$ 引物X2 (20 $\mu \mathrm{M}), 36.5 \mu \mathrm{L}$ 灭菌蒸馏水。PCR反应条件为 $95^{\circ} \mathrm{C} 5$ $\min ; 95^{\circ} \mathrm{C} 30 \mathrm{~s}, 54^{\circ} \mathrm{C} 30 \mathrm{~s}, 72^{\circ} \mathrm{C} 30 \mathrm{~s}$, 共30个循环; $72^{\circ} \mathrm{C} 10 \mathrm{~min} ; 16^{\circ} \mathrm{C} 10 \mathrm{~min}$ 。将纯化后质量合格的 PCR产物进行DNA文库的构建。建库成功后挑取克 隆子, 利用Illumina公司 Miseq台式测序仪 $2 \times 250$ bp双末端测序法进行测序。

\section{3 高通量测序数据处理与统计分析}

高通量测序得到的原始数据经过 FastQC (http://www.bioinformatics.babraham.ac.uk/projects/f astqc/) 质量控制, 然后用 FLASH (http://ccb.jhu. edu/software/FLASH/) (Magoč \& Salzberg, 2011)将 有overlap的一对双末端reads进行拼接, 过滤掉低质 量的序列。使用QIIME (版本1.8.0)(Caporaso et al., 2010)将样品标准化为每样品36,000条序列, 依据惯 例(Stackebrandt \& Goebel，1994)将相似性不低于 $97 \%$ 的序列聚类成为 OTU (operational taxonomic unit)。为了高效地得到样品中微生物组成的多样性 信息, 我们从每个OTU中选择1条代表序列, 使用 RDP classifier (版本 2.2)(Cole et al., 2014)依据 GreenGenes数据库为每条代表序列分配分类单元; 再用QIIME (Caporaso et al., 2010)进行单样品组成 分析, 得到样品在不同分类水平上的细菌类群组成 及相对丰度的数据, 将其中无法鉴定到这个分类水 平的类群合并统称为未知类群(Unknown), 将相对 丰度低于 $1 \%$ 的类群合并统称为其他类群(Others), 然后制作不同分类水平上的细菌优势类群相对丰 度图。

在上述基础上制作采样点数-土壤细菌类群数 曲线。具体的做法是: 从 31 个样品中随机抽取 1 个样 品, 记下这个样品中包含的细菌类群数目; 然后从 剩下的 30 个样品中再随机抽取 1 个样品数据, 和第 一个抽到的样品数据合并, 记下这 2 个样品中包含 的细菌类群数目; 依此类推, 直至采样点的数目达 到最大; 重复99次取平均值, 制作采样点数-土壤 细菌类群曲线。

本研究其他的数据分析与图形制作使用 R 3.1.2 软件(R-Development Core Team, 2014)完成。

\section{2 结果}

\section{1 高通量测序原始数据处理}

测序原始数据为两端各得到10,223,657条reads, 拼接并进行预处理、质控后剩余9,530,983条序列。 预设每个样品 40,000条序列, 然后去除嵌合体, 去 除Singleton (只有1条序列的那些OTU), 并标准化 为每样品 36,000 条序列, 得到每个样品的序列数及 OTU数(表1)。

\section{2 土壤细菌分类}

对宝天曼样地31个土壤样品进行高通量测序, 共产生42,632个OTU。与数据库比对不上的OTU即 未分配的部分(Unassigned)约占总数的 $0.76 \%$ 。31个 土壤样品中平均有 $5,924.7 \pm 94.9$ 个OTU, 其中单个 样品最多的有 6,979 个、最少的有 4,971 个，分属于 45 门163纲319目495科785属，细菌分类群详细信息见 附录1(不包含未分配的部分; 在每个分类水平上未 有明确分类名称信息的也算作 1 个计入其中)。

\subsection{1 门}

31 个土壤样品中平均有细菌34.2 \pm 0.40 门, 其 中单个样品最多的有39门，最少的有30门(图2)。检 
表1 样品序列数及OTU数

Table 1 Sequence numbers and OTU numbers of individual samples

\begin{tabular}{|c|c|c|c|c|}
\hline $\begin{array}{l}\text { 样品编号 } \\
\text { Sample } \\
\text { identity }\end{array}$ & $\begin{array}{c}\text { 原始序列 } \\
\text { 数 } \\
\text { Original } \\
\text { sequence } \\
\text { number }\end{array}$ & $\begin{array}{c}\text { 原始OTU数 } \\
\text { Original OTU } \\
\text { number }\end{array}$ & $\begin{array}{c}\text { 标准化序列数 } \\
\text { Even sequence } \\
\text { number }\end{array}$ & $\begin{array}{c}\text { 标准化后 } \\
\text { OTU数 } \\
\text { Even OTU } \\
\text { number }\end{array}$ \\
\hline 0101 & 37,569 & 7,148 & 36,000 & 6,979 \\
\hline 0105 & 39,949 & 5,671 & 36,000 & 5,320 \\
\hline 0203 & 39,698 & 5,661 & 36,000 & 5,327 \\
\hline 0218 & 39,337 & 5,579 & 36,000 & 5,320 \\
\hline 0306 & 39,281 & 6,396 & 36,000 & 6,097 \\
\hline 0318 & 39,760 & 6,263 & 36,000 & 5,906 \\
\hline 0411 & 39,756 & 6,008 & 36,000 & 5,672 \\
\hline 0606 & 39,636 & 6,642 & 36,000 & 6,286 \\
\hline 0701 & 39,945 & 6,059 & 36,000 & 5,694 \\
\hline 0819 & 39,864 & 6,324 & 36,000 & 5,933 \\
\hline 0913 & 39,827 & 5,275 & 36,000 & 4,971 \\
\hline 0919 & 39,720 & 5,958 & 36,000 & 5,645 \\
\hline 1002 & 39,844 & 5,486 & 36,000 & 5,175 \\
\hline 1006 & 39,551 & 6,658 & 36,000 & 6,322 \\
\hline 1018 & 39,711 & 5,526 & 36,000 & 5,184 \\
\hline 1104 & 39,746 & 6,380 & 36,000 & 6,036 \\
\hline 1210 & 39,569 & 6,255 & 36,000 & 5,933 \\
\hline 1212 & 39,659 & 6,016 & 36,000 & 5,691 \\
\hline 1318 & 39,809 & 6,706 & 36,000 & 6,326 \\
\hline 1405 & 39,825 & 5,910 & 36,000 & 5,547 \\
\hline 1510 & 39,379 & 5,650 & 36,000 & 5,378 \\
\hline 1512 & 37,588 & 5,555 & 36,000 & 5,413 \\
\hline 1607 & 39,739 & 6,338 & 36,000 & 5,981 \\
\hline 1615 & 39,212 & 6,246 & 36,000 & 5,978 \\
\hline 1618 & 39,578 & 6,552 & 36,000 & 6,200 \\
\hline 1704 & 39,495 & 6,405 & 36,000 & 6,024 \\
\hline 1718 & 39,130 & 6,910 & 36,000 & 6,564 \\
\hline 1808 & 39,739 & 6,833 & 36,000 & 6,498 \\
\hline 1814 & 39,866 & 6,850 & 36,000 & 6,501 \\
\hline 1902 & 39,155 & 7,283 & 36,000 & 6,920 \\
\hline 1913 & 38,882 & 7,145 & 36,000 & 6,844 \\
\hline
\end{tabular}

测到的 45 个门中有 42 个有明确分类信息， 1 个分类 信息有争议, 2 个未有明确分类名称信息。相对丰度 最高的 5 个门所占比例总和达到 $82.34 \%$, 分别是变 形菌门(Proteobacteria)(38.30\%)、酸杆菌门(Acidobacteria)(24.57\%)、疮微菌门 (Verrucomicrobia) (9.98\%)、放线菌门(Actinobacteria)(6.10\%)和浮霉菌 门(Planctomycetes)(3.38\%)(附录2A)。

\subsection{2 纲}

31 个土壤样品中平均有细菌 $114.7 \pm 1.4$ 纲, 其 中单个样品最多的有131纲, 最少的有 97 纲(图2)。检
测到的 163 个纲中有 120 个有明确分类信息，8个分 类信息有争议, 35个未有明确分类名称信息。相对 丰度最大的 5 个纲所占比例总和达到 $50.93 \%$ ，分别 是 $\alpha$-变形菌纲 $(\alpha$-Proteobacteria) $(18.08 \%) 、 D A 052$ $(9.55 \%) 、 S p a r t o b a c t e r i a(8.44 \%) 、 \beta$-变形菌纲 $(\beta-$ Proteobacteria) $(7.77 \%)$ 和 $\gamma$ - 变形菌纲 $(\gamma$-Proteobacteria) (7.10\%) (附录2B)。

\subsection{3 目}

31 个土壤样品中平均有细菌 $215.2 \pm 2.8$ 目, 其 中单个样品最多的有 250 目，最少的187目(图2)。检 测到的 319 个目中有 181 个有明确分类信息，10个分 类信息有争议, 128 个未有明确分类名称信息。相对 丰度最大的 5 个目所占比例总和达到 $40.05 \%$, 分别 是根瘤菌目 (Rhizobiales)(10.62\%)、Ellin6513 (9.55\%)、Chthoniobacterales (8.44\%)、酸杆菌目(Acidobacteriales) (6.76\%)和黄单胞菌目 (Xanthomonadales) (4.69\%)(附录2C)。

\subsection{4 科}

31 个土壤样品中平均有细菌 $323.7 \pm 3.8$ 科, 其 中单个样品最多的有 370 科, 最少的有288科(图2)。 检测到的 495 个科中有 191 个有明确分类信息，14个 分类信息有争议, 290 个未有明确分类名称信息。相 对丰度最大的 5 个科所占比例总和达到 $34.05 \%$, 分 别是Ellin6513目某科(Ellin6513; f__)(9.55\%)、Chthoniobacteraceae $(8.44 \%) 、$ 生丝微菌科(Hyphomicrobiaceae)(6.16\%)、Koribacteraceae (6.00\%)和中华杆 菌科(Sinobacteraceae)(3.90\%)(附录2D)。

\subsection{5 属}

31 个土壤样品中平均有细菌 $446.6 \pm 5.4$ 属, 其 中单个样品最多的有511属, 最少的398属(图2)。检 测到的 785 个属中有 261 个有明确分类信息, 524 个 未有明确分类名称信息。相对丰度最大的 5 个属所 占比例总和达到 $30.87 \%$, 分别是Ellin6513目某科某 属 (Ellin6513; f_; g_)(9.55\%)、DA101 (7.09\%)、 Koribacteraceae 科某属 (Koribacteraceae; g_ ) (5.50\%)、红游动菌属(Rhodoplanes)(4.89\%)和红螺菌 科某属(Sinobacteraceae; g__(3.85\%)(附录2E)。

\section{3 取样数量对土壤细菌多样性的影响}

在不同的分类水平上，随着取样点的增加，检 测出的土壤细菌类群的数目也在增加。当取样点达 到31个时, 检测出的土壤细菌类群的数目达到最 大，分别为45门 163 纲 319 目 495 科785属42,632个 


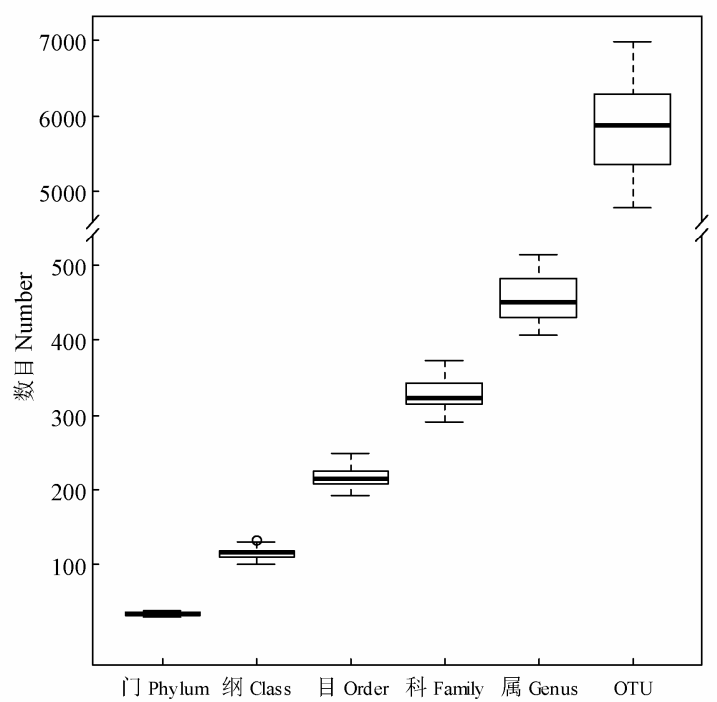

图2 宝天曼森林动态监测样地 31 个土壤样品中检测到的不 同分类水平上的细菌数量

Fig. 2 The quantity of bacteria at different taxonomic levels in the 31 soil samples in the Baotianman Forest Dynamics Plot

\section{OTU (图3)。}

在门的分类水平上，取样点数在1-10区间时， 检测到的细菌门数量随着取样点数量增加而快速 增加; 取样点数超过 10 后, 门的数量增速减慢; 当 取样点数为 19 个时, 约能检测到 44 个门; 取样点数 在20-31区间时, 门数仅增加 1 个达到 45 个。

在纲的分类水平上，取样点数在1-10区间时， 检测到的纲数量随着取样点数量增加而较快增加; 当取样点数超过 10 后, 纲数量增速减慢; 当取样点 数为 27 个时, 检测到约 162 个细菌纲; 取样点数在 27-31区间时, 纲数仅增加 1 个, 达到 163 个。

在目、科和属的分类水平上, 取样点数在 $1-10$ 区间时, 检测到的相应细菌类群数量随着取样点数 量增加而较快增加; 当取样点数超过 10 后, 细菌类 群数目增速减慢、但增速仍较大, 且属的增速大于科 的增速、科的增速大于目的增速。在OTU的分类水 平上, 取样点数在1-31区间时, 检测到的细菌OTU 数量随着取样点数量增加一直在较快增加(图3)。

\section{3 讨论}

本文采用Illumina公司Miseq测序仪对地处南暖 温带向北亚热带过渡区的宝天曼落叶阔叶林土壤 细菌多样性进行了初步研究, 列出了宝天曼森林土
壤细菌门、纲、目、科、属、OTU数量以及不同分 类水平上的优势类群及其相对丰度。虽然本次调查 采集的土壤样品数量有限, 但高通量测序技术的应 用为进一步调查宝天曼样地内土壤细菌等微生物 资源积累了经验，并提供了新的技术支撑。在本研 究中, 与数据库比对不上的OTU约占总数的 $0.76 \%$, 这说明所参考的GreenGenes数据库中包含了宝天 曼森林土壤细菌类群中的绝大部分。研究显示, 宝 天曼森林土壤细菌在门和纲水平上的分类信息较 明确(附录 $2 \mathrm{~A}, \mathrm{~B}$ ), 而在目、科和属的水平上尚无明 确分类名称信息的类群所占比例较大 (附录 $2 \mathrm{C}-\mathrm{E}$ ), 这可能与测序区间、序列长度以及比对的数据库等 有关。

本研究在宝天曼森林土壤中共检测出变形菌 门、酸杆菌门、疮微菌门等45门, 变形菌门是最丰 富的细菌类群, 这一结果与国内外关于农田、森林 和草地土壤细菌多样性的很多研究相一致(滕齐辉 等, 2006; 张建萍等, 2008; Nacke et al., 2011; Shen et al., 2013)。宝天曼样地植被类型主要为落叶阔叶 林, Zhang等(2014b)对湖北神农架的落叶阔叶林研 究中也检测出变形菌门是含量最丰富的类群, 这可 能说明林型也是影响土壤细菌群落的因素。Fierer 等(2012)发现在温带森林中酸杆菌是土壤中含量最 丰富的细菌类群, Yuan等(2014)在我国青藏草地的 研究中也发现酸杆菌是土壤中含量最丰富的细菌 类群, 而本研究中, 酸杆菌门含量次于变形菌门居 第二位, 这也验证了杨官品等(2000)的观点：土壤 细菌具有非常丰富的多样性, 不同类型的土壤中存 在各自的优势细菌类群, 同时又有相似的细菌 类群。

已有土壤细菌或土壤微生物研究中采用的取 样数量变化很大(如 1 个、3个、 10 个、36个样品等), 但 具体到某种植被类型、生境类型或生态系统, 需要 采集分析多少土壤样品才能较好地反映其微生物 多样性水平，仍是个没有很好回答的问题。值得注 意的是, 在采样数量一定的情况下, 研究人员大多 还采取把数个取样点土样混合后形成混合样品以 增加样品的代表性(Fierer \& Jackson, 2006; Jones et al., 2009; Bates et al., 2010; Li et al., 2014)。我们的 初步研究表明, 取样数量对检测出的土壤细菌类群 数量有显著影响: 随着样品数量增多, 被检测出来 的细菌类群数量也增多, 当达到 31 个样品时, 其增 

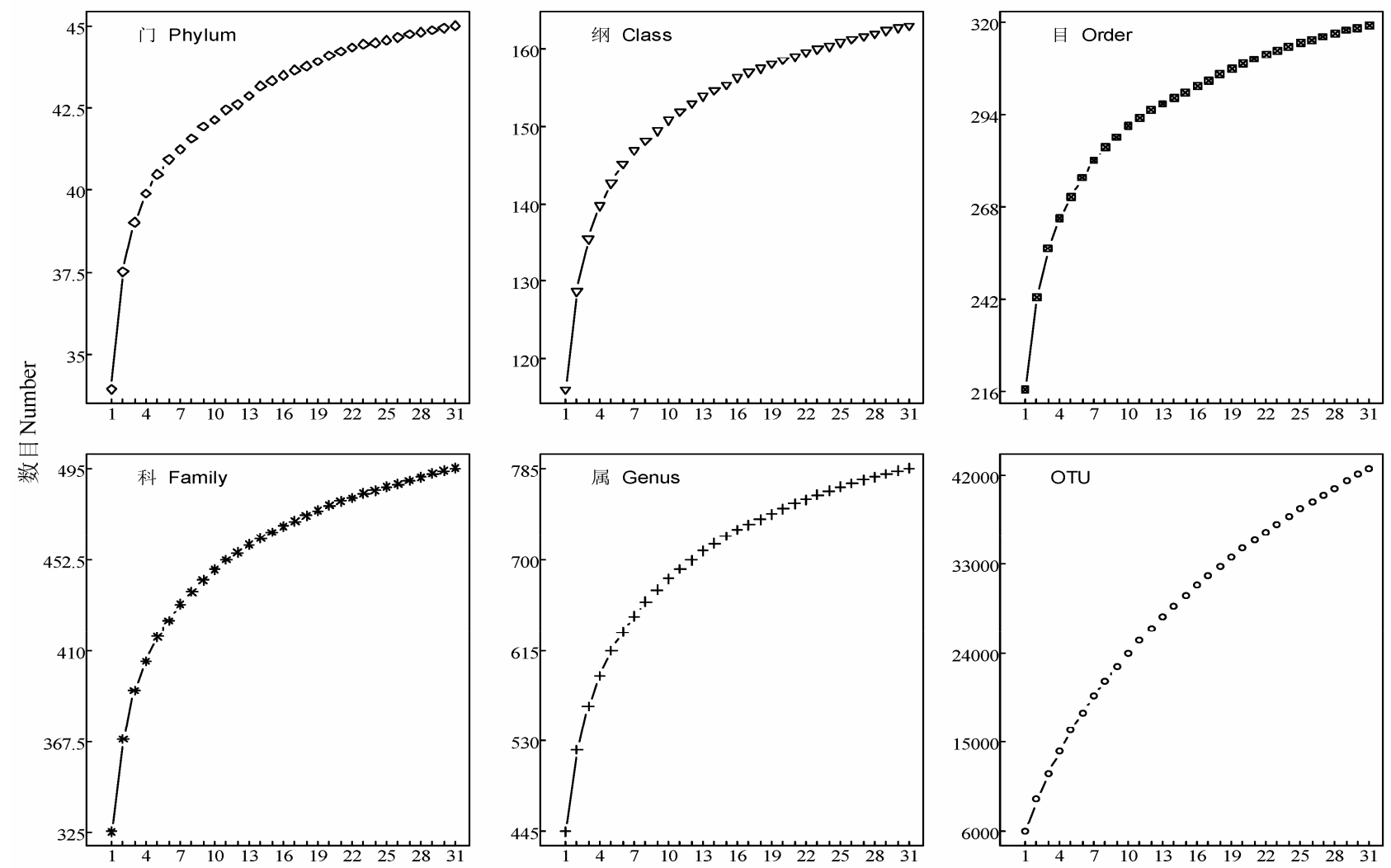

图3 宝天曼森林动态监测样地31个土壤样品中检测到的不同分类水平上细菌类群数随采样点数增加的稀疏曲线

Fig. 3 Rarefaction curves of the observed bacterial quantity at different taxonomic levels varying with number of samples in the Baotianman Forest Dynamics Plot

速虽然趋缓但仍在上升, 且不同分类水平的细菌类 群多样性表现出类似趋势同时也有区别(图3), 这说 明增加土壤采样点数量应能检测出更多的细菌类 群, 同时也提醒我们在对不同研究比较时要注意取 样数量的影响。本研究结果显示无论是哪个分类水 平, 当取样点数在1-10区间时, 检测到的细菌类群 数量均随着取样点数量增加而增加较快, 这说明当 取样数量较少时可能会有较多细菌类群没有检测 出来。在宝天曼森林中, 在门的分类水平上, 19个样 品基本可以代表31个样品检测出来的细菌门多样 性信息; 在纲的分类水平上, 27 个样品基本可以代 表31个样品检测出来的细菌纲多样性信息; 在目、

科、属的分类水平上当样品数量达到 31 个时检测出 来的相应细菌类群数仍在逐渐增加; 特别是在OTU 的水平上当样品数量达到 31 个时细菌OTU数量仍 在较快速增加, 推测如果增加较多样品数量, 检测 出来的细菌OTU数量还会有较大增加。此问题需要
进一步深入研究。

具体研究中, 无论是草地(Nacke et al., 2011; Yuan et al., 2014)、农田(Shi et al., 2010; Liu et al., 2013)还是在森林(Hackl et al., 2004; Jiang et al., 2010; Li et al., 2014)等的土壤微生物研究, 均应重 视取样数量对研究结果的影响。取样数量除了受研 究目的、研究精度决定外, 往往也受实验研究成本 影响, 特别是高通量测序目前的单个样品所需费用 还较高的情况下。因此, 在实际研究中往往需要权 衡选择一定取样数量, 既能满足研究目的和反映研 究对象的微生物多样性水平, 又能兼顾实验研究费 用。

另外, 文库的构建及测序深度的选择等也会对 检测出的细菌类群产生影响。本研究测序深度为每 样品36,000条序列, 文库的覆盖率为 $90.48 \%$, 如果 增加库容、加大测序深度, 有望检测出更多的细菌 类群。再者, 测序区间的选择也会对结果有一定影 
响, 如16S rRNA基因的高变区不同, 其分类信息的 精确性也不同(Chakravorty et al., 2007)。

Nacke等(2011)在德国的研究表明, 土壤细菌群 落的结构在很大程度上是受树种和土壤 $\mathrm{pH}$ 值影响 的; 在不同的空间尺度上, $\mathrm{pH}$ 值对土壤细菌的多样 性和群落组成均有很大的影响(Lauber et al., 2009; Rousk et al., 2010; Griffiths et al., 2011; Fierer et al., 2012)。土壤化学因子、土壤温度、植物多样性是形 成森林土壤微生物群落结构的3个主要因素(Zhang et al., 2014b)。森林类型也可能是影响森林土壤微生 物群落组成的因素之一。是哪些环境因子影响着宝 天曼森林土壤细菌群落的组成和结构? 土壤细菌 不同类群有什么样的功能? 土壤细菌与其他生物 类群之间是如何相互作用的? 土壤细菌多样性与 植物多样性的关系是怎样的? 土壤细菌在森林群 落维持上扮演着什么样的角色等? 这些问题都有 待进一步的研究。

致谢: 感谢马克平研究员对本研究的帮助, 感谢间 满玉和南阳师范学院生物系部分同学在野外土壤 采样、白淑华和于冬梅在土壤样品DNA提取、刘威 在数据分析中的帮助, 感谢宝天曼国家级自然保护 区在野外工作中的支持和帮助。

\section{参考文献}

Bates ST, Berg-Lyons D, Caporaso JG, Walters WA, Knight R, Fierer N (2010) Examining the global distribution of dominant archaeal populations in soil. The International Society for Microbial Ecology, 5, 908-917.

Cai CQ (蔡晨秋), Tang L (唐丽), Long CL (龙春林) (2011) Soil microbial diversity and its research methods. Journal of Anhui Agricultural Science (安徽农业科学), 39, 1727417276, 17278. (in Chinese with English abstract)

Caporaso JG, Kuczynski J, Stombaugh J, Bittinger K, Bushman FD, Costello EK, Fierer N, Peña AG, Goodrich JK, Gordon JI, Huttley GA, Kelley ST, Knights D, Koenig JE, Ley RE, Lozupone CA, McDonald D, Muegge BD, Pirrung M, Reeder J, Sevinsky JR, Turnbaugh PJ, Walters WA, Widmann J, Yatsunenko T, Zaneveld J, Knight R (2010) QIIME allows analysis of high-throughput community sequencing data. Nature Methods, 7, 335-336.

Caporaso JG, Lauber CL, Walters WA, Berg-Lyons D, Lozupone CA, Turnbaugh PJ, Fierer N, Knight R (2011) Global patterns of 16S rRNA diversity at a depth of millions of sequences per sample. Proceedings of the National Academy of Sciences, USA, 108 (S1), 4516-4522.

Caporaso JG, Lauber CL, Walters WA, Berg-Lyons D, Huntley J, Fierer N, Owens SM, Betley J, Fraser L, Bauer M, Gorm- ley N, Gilbert JA, Smith G, Knight R (2012) Ultra-high-throughput microbial community analysis on the Illumina Hiseq and Miseq platforms. The International Society for Microbial Ecology, 6, 1621-1624.

Chakravorty S, Helb D, Burday M, Connell N, Allan D (2007) A detailed analysis of $16 \mathrm{~S}$ ribosomal RNA gene segments for the diagnosis of pathogenic bacteria. Journal of Microbiological Methods, 69, 330-339.

Cole JR, Wang Q, Fish JA, Chai B, McGarrell DM, Sun Y, Brown CT, Porras-Alfaro A, Kuske CR, Tiedje JM (2014) Ribosomal Database Project: data and tools for high throughput rRNA analysis. Nucleic Acids Research, 42 (Datbase issue), D633-D642.

Delmont TO, Prestat E, Keegan KP, Faubladier M, Robe P, Clark IM, Pelletier E, Hirsch PR, Meyer F, Gilbert JA, Le Paslier D, Simonet P, Vogel TM (2012) Structure, fluctuation and magnitude of a natural grassland soil metagenome. The International Society for Microbial Ecology, 6, 1677-1687.

Fernando WJD, Li R (2012) Opening the black box: understanding the influence of cropping systems and plant communities on bacterial and fungal population dynamics. Ceylon Journal of Science (Biology Science), 41, 89-110.

Fierer N, Jackson RB (2006) The diversity and biogeography of soil bacterial communities. Proceedings of the National Academy of Sciences, USA, 103, 626-631.

Fierer N, Leff JW, Adams BJ, Nielsend UN, Thomas BS, Lauber CL, Sarah O, Gilberte JA, Wall DH, Caporaso JG (2012) Cross-biome metagenomic analyses of soil microbial communities and their functional attributes. Proceedings of the National Academy of Sciences, USA, 109, 21390-21395.

Griffiths RI, Thomson BC, James P, Bell T, Bailey M, Whiteley AS (2011) The bacterial biogeography of British soils. Environmental Microbiology, 13, 1642-1654.

Gu FX (顾峰雪), Wen QK (文启凯), Pan BR (潘伯荣), Yang YS (杨玉锁) (2000) A preliminary study on soil microorganisms of artificial vegetation in the center of Taklimakan Desert. Biodiversity Science (生物多样性), 8, 297-303. (in Chinese with English abstract)

Guo LD (郭良栋) (2012) Progress of microbial species diversity research in China. Biodiversity Science (生物多样性), 20, 572-580. (in Chinese with English abstract)

Hackl E, Pfeffer M, Donat C, Bachmann G, Zechmeister-Boltenstern S (2005) Composition of the microbial communities in the mineral soil under different types of natural forest. Soil Biology and Biochemistry, 37, 661-671.

Hackl E, Zechmeister-Boltenstern S, Bodrossy L, Sessitsch A (2004) Comparison of diversities and compositions of bacterial populations inhabiting natural forest soils. Applied Environmental Microbiology, 70, 5057-5065.

He ZL, van Nostrand JD, Deng Y, Zhou JZ (2011) Development and applications of functional gene microarrays in the analysis of the functional diversity, composition, and structure of microbial communities. Frontiers of Environmental Science and Engineering in China, 5, 1-20. 
Jiang HY (姜海燕), Yan W (间伟), Li XT (李晓泮), Yang XL (杨秀丽), Lv HL (吕洪丽) (2010) The diversity of soil microorganism under different vegetations of Larix gmelinii forest in Great Xing'an Mountains. Microbiology China (微 生物学通报), 37, 186-190. (in Chinese with English abstract)

Jones RT, Robeson MS, Lauber CL, Hamady M, Knight R, Fierer N (2009) A comprehensive survey of soil acidobacterial diversity using pyrosequencing and clone library analyses. The International Society for Microbial Ecology, 3, 442-453.

Lauber CL, Micah H, Knight R, Fierer N (2009) Pyrosequencing-based assessment of soil $\mathrm{pH}$ as a predictor of soil bacterial community structure at the continental scale. Applied and Environmental Microbiology, 75, 5111-5120.

Li H, Ye D, Wang X, Settles ML, Wang J, Hao Z, Zhou L, Dong P, Jiang Y, Ma ZS (Sam) (2014) Soil bacterial communities of different natural forest types in Northeast China. Plant Soil, 383, 203-216.

Liu YD (刘雨迪), Chen XY (陈小云), Liu MQ (刘满强), Qin JT (秦江涛), Li HX (李辉信), Hu F (胡锋) (2013) Changes in soil microbial properties and nematode assemblage over time during rice cultivation. Biodiversity Science (生物多样 性), 21, 334-342. (in Chinese with English abstract)

Macrae A (2000) The use of 16S rDNA methods in soil microbial ecology. Brazilian Journal of Microbiology, 31, 77-82.

Magoč T, Salzberg SL (2011) FLASH: fast length adjustment of short reads to improve genome assemblies. Bioinformatics, 27, 2957-2963.

Nacke H, Thürmer A, Wollherr A, Will C, Hodac L, Herold N, Schöning I, Schrumpf M, Daniel R (2011) Pyrosequencing-based assessment of bacterial community structure along different management types in German forest and grassland soils. PLOS ONE, 6, e17000.

Roesch LFW, Fulthorpe RR, Riva A, Casella G, Hadwin AKM, Kent AD, Daroub SH, Camargo FAO, FarmerieWG, Triplett EW (2007) Pyrosequencing enumerates and contrasts soil microbial diversity. The International Society for Microbial Ecology, 1, 283-290.

Rousk J, Bååth E, Brookes PC, Lauber CL, Lozupone C, Caporaso JG, Knight R, Fierer N (2010) Soil bacterial and fungal communities across a $\mathrm{pH}$ gradient in an arable soil. The International Society for Microbial Ecology, 4, 1340-1351.

Shen CC, Xiong JB, Zhang HY, Feng YZ, Lin XG, Li XY, Liang WJ, Chu HY (2013) Soil pH drives the spatial distribution of bacterial communities along elevation on Changbai Mountain. Soil Biology and Biochemistry, 57, 204-211.

Shi P (时鹏), Gao Q (高强), Wang SP (王淑平), Zhang Y (张 妍) (2010) Effects of continuous cropping of corn and fertilization on soil microbial community functional diversity. Acta Ecologica Sinica (生态学报), 30, 6173-6182. (in Chinese with English abstract)

Song CS (宋朝枢) (1994) Scientific Investigation in the Bao- tianman Nature Reserve (宝天曼自然保护区科学考察集). China Forestry Publishing House, Beijing. (in Chinese)

Stackebrandt E, Goebel BM (1994) Taxonomic note: a place for DNA-DNA reassociation and 16S rRNA sequence analysis in the present species definition in bacteriology. International Journal of Systematic Bacteriology, 44, 846-849.

Sun X (孙欣), Gao Y (高芗), Yang YF (杨云锋) (2013) Recent advancement in microbial environmental research using metagenomics tools. Biodiversity Science (生物多样性), 21, 393-400. (in Chinese with English abstract)

Teng QH (滕齐辉), Cao H (曹慧), Cui ZL (崔中利), Wang Y (王英), Sun B (孙波), Hao HT (郝红涛), Li SP (李顺鹏) (2006) PCR-RFLP analysis of bacterial 16S rDNA from a typical garden soil in Taihu region. Biodiversity Science (生 物多样性), 14, 345-351. (in Chinese with English abstract)

Tiedje JM, Asuming-Brempong S, Nüsslein K, Marsh TL, Flynn SJ (1999) Opening the black box of soil microbial diversity. Applied Soil Ecology, 13, 109-122.

van der Heijden MGA, Bardgett RD, van Straalen NM (2008) The unseen majority: soil microbes as drivers of plant diversity and productivity in terrestrial ecosystems. Ecology Letters, 11, 296-310.

Wooley JC, Godzik A, Friedberg I (2010) A primer on metagenomics. PLoS Computational Biology, 6, e1000667.

Wu ZY (吴则焰), Lin WX (林文雄), Chen ZF (陈志芳), Fang CX (方长旬), Zhang ZX (张志兴), Wu LK (吴林坤), Zhou MM (周明明), Chen T (陈婷) (2013) Variations of soil microbial community diversity along an elevational gradient in mid-subtropical forest. Chinese Journal of Plant Ecology (植物生态学报), 37, 397-406. (in Chinese with English abstract)

Yang GP (杨官品), Nan L (男兰), Jia HB (贾海波), Zhu YH (朱艳红), Liu YJ (刘英杰), Zhang K (张凯) (2000) Bacterial genetic diversity in soils and their correlation with vegetation. Acta Genetica Sinica (遗传学报), 27, 278-282. (in Chinese with English abstract)

Yuan YL, Si GC, Wang J, Luo TX, Zhang GX (2014) Bacterial community in alpine grasslands along an altitudinal gradient on the Tibetan Plateau. FEMS Microbiology Ecology, 87, 121-132.

Zhang JP (张建萍), Dong NY (董乃源), Yu HB (余浩滨), Zhou YJ (周勇军), Lu YL (陆永良), Geng RM (耿锐梅), Yu LQ (余柳青) (2008) Bacteria diversity in paddy field soil by $16 \mathrm{~S}$ rDNA-RFLP analysis in Ningxia. Biodiversity Science (生物多样性), 16, 586-592. (in Chinese with English abstract)

Zhang YG, Cong J, Lu H, Li GL, Qu YY, Su XJ, Zhou JZ, Li DQ (2014a) Community structure and elevational diversity patterns of soil Acidobacteria. Journal of Environmental Sciences, 26, 1717-1724.

Zhang YG, Cong J, Lu H, Yang CY, Yang YF, Zhou JZ, Li DQ (2014b) An integrated study to analyze soil microbial community structure and metabolic potential in two forest types. 
PLOS ONE, 9, e93773.

Zhao XL (赵先丽), Zhou GS (周广胜), Zhou L (周莉), Lv GH

(吕国红), Jia QY (贾庆宇), Xie YB (谢艳兵) (2007) A preliminary study on soil microorganism in Panjin reed wetland. Journal of Meteorology and Environment (气象与 环境学报), 23, 30-33. (in Chinese with English abstract)

(责任编委: 贺纪正 责任编辑: 时意专)

\section{附录 Supplementary Material}

附录1 宝天曼森林动态监测样地土壤细菌名录(-表示相应分类水平上没有明确分类名称信息)

Appendix 1 Soil bacteria list in the Baotianman Forest Dynamics Plot. - means without specific names at the relative classification levels.

http://www.biodiversity-science.net/fileup/PDF/w2015-032-1.pdf

附录2 宝天曼森林动态监测样地31个土壤样品中检测到的不同分类水平上细菌优势类群及其相对丰度(其中Unknown代表 未鉴定出的类群, Others代表丰度低于1\%的类群)

Appendix 2 Relative abundances of the dominant bacteria at different taxonomic levels in the 31 soil samples in the Baotianman Forest Dynamics Plot. Unknown means the taxa that can not be identified; Others represent the taxa whose relative abundances are less than $1 \%$.

http://www.biodiversity-science.net/fileup/PDF/w2015-032-2.pdf 
附录1 宝天曼森林动态监测样地土壤细菌名录(-表示相应分类水平上没有明确分类名称信息)

Appendix 1 Soil bacteria list in the Baotianman Forest Dynamics Plot. - means without specific names at the relative classification levels

\begin{tabular}{|c|c|c|c|c|}
\hline 门 Phylum & 纲 Class & 目 Order & 科 Family & 属 Genus \\
\hline Planctomycetes & Planctomycetia & Pirellulales & Pirellulaceae & A17 \\
\hline Proteobacteria & Betaproteobacteria & Burkholderiales & Alcaligenaceae & Achromobacter \\
\hline Proteobacteria & Alphaproteobacteria & Rhodospirillales & Acetobacteraceae & Acidiphilium \\
\hline Proteobacteria & Alphaproteobacteria & Rhodospirillales & Acetobacteraceae & Acidisoma \\
\hline Proteobacteria & Alphaproteobacteria & Rhodospirillales & Acetobacteraceae & Acidocella \\
\hline Proteobacteria & Betaproteobacteria & Burkholderiales & Comamonadaceae & Acidovorax \\
\hline Proteobacteria & Gammaproteobacteria & Pseudomonadales & Moraxellaceae & Acinetobacter \\
\hline Actinobacteria & Actinobacteria & Actinomycetales & Streptosporangiaceae & Acrocarpospora \\
\hline Actinobacteria & Actinobacteria & Actinomycetales & Thermomonosporaceae & Actinoallomurus \\
\hline Actinobacteria & Actinobacteria & Actinomycetales & Thermomonosporaceae & Actinocorallia \\
\hline Actinobacteria & Actinobacteria & Actinomycetales & Thermomonosporaceae & Actinomadura \\
\hline Actinobacteria & Actinobacteria & Actinomycetales & Pseudonocardiaceae & Actinomycetospora \\
\hline Actinobacteria & Actinobacteria & Actinomycetales & Micromonosporaceae & Actinoplanes \\
\hline Bacteroidetes & Cytophagia & Cytophagales & Cytophagaceae & Adhaeribacter \\
\hline Actinobacteria & Actinobacteria & Actinomycetales & Nocardioidaceae & Aeromicrobium \\
\hline Proteobacteria & Alphaproteobacteria & Rhizobiales & Rhodobiaceae & Afifella \\
\hline Proteobacteria & Alphaproteobacteria & Rhizobiales & Bradyrhizobiaceae & Afipia \\
\hline Proteobacteria & Alphaproteobacteria & Rhizobiales & Rhizobiaceae & Agrobacterium \\
\hline Actinobacteria & Actinobacteria & Actinomycetales & Microbacteriaceae & Agromyces \\
\hline Firmicutes & Bacilli & Bacillales & Alicyclobacillaceae & Alicyclobacillus \\
\hline Proteobacteria & Gammaproteobacteria & Pseudomonadales & Moraxellaceae & Alkanindiges \\
\hline Proteobacteria & Alphaproteobacteria & Rhodobacterales & Rhodobacteraceae & Amaricoccus \\
\hline Proteobacteria & Alphaproteobacteria & Rhizobiales & Phyllobacteriaceae & Aminobacter \\
\hline Firmicutes & Bacilli & Bacillales & Paenibacillaceae & Ammoniphilus \\
\hline Actinobacteria & Actinobacteria & Actinomycetales & Pseudonocardiaceae & Amycolatopsis \\
\hline Chloroflexi & Anaerolineae & Anaerolineales & Anaerolinaceae & Anaerolinea \\
\hline Proteobacteria & Deltaproteobacteria & Myxococcales & Myxососсасеаe & Anaeromyxobacter \\
\hline Firmicutes & Bacilli & Bacillales & Bacillaceae & Anoxybacillus \\
\hline Proteobacteria & Gammaproteobacteria & Legionellales & Coxiellaceae & Aquicella \\
\hline Actinobacteria & Actinobacteria & Actinomycetales & Micrococcaceae & Arthrobacter \\
\hline Tenericutes & Mollicutes & Anaeroplasmatales & Anaeroplasmataceae & Asteroleplasma \\
\hline Proteobacteria & Alphaproteobacteria & Caulobacterales & Caulobacteraceae & Asticcacaulis \\
\hline Proteobacteria & Betaproteobacteria & Burkholderiales & Comamonadaceae & Azohydromonas \\
\hline Proteobacteria & Betaproteobacteria & Rhodocyclales & Rhodocyclaceae & Azospira \\
\hline Firmicutes & Bacilli & Bacillales & Bacillaceae & Bacillus \\
\hline Proteobacteria & Deltaproteobacteria & Bdellovibrionales & Bacteriovoracaceae & Bacteriovorax \\
\hline Proteobacteria & Alphaproteobacteria & Rhizobiales & Bradyrhizobiaceae & Balneimonas \\
\hline Proteobacteria & Deltaproteobacteria & Bdellovibrionales & Bdellovibrionaceae & Bdellovibrio \\
\hline Proteobacteria & Alphaproteobacteria & Rhizobiales & Xanthobacteraceae & Blastochloris \\
\hline
\end{tabular}




\begin{tabular}{|c|c|c|c|c|}
\hline 门 Phylum & 纲 Class & 目 Order & 科 Family & 属 Genus \\
\hline Proteobacteria & Alphaproteobacteria & Sphingomonadales & Sphingomonadaceae & Blastomonas \\
\hline Proteobacteria & Alphaproteobacteria & Rhizobiales & Bradyrhizobiaceae & Bosea \\
\hline Proteobacteria & Betaproteobacteria & Burkholderiales & Comamonadaceae & Brachymonas \\
\hline Proteobacteria & Alphaproteobacteria & Rhizobiales & Bradyrhizobiaceae & Bradyrhizobium \\
\hline Firmicutes & Bacilli & Bacillales & Paenibacillaceae & Brevibacillus \\
\hline Proteobacteria & Betaproteobacteria & Burkholderiales & Burkholderiaceae & Burkholderia \\
\hline Chloroflexi & Anaerolineae & Caldilineales & Caldilineaceae & Caldilinea \\
\hline Firmicutes & Clostridia & Clostridiales & Clostridiaceae & Caloramator \\
\hline Bacteroidetes & Cytophagia & Cytophagales & [Amoebophilaceae] & Candidatus Amoebophilus \\
\hline Proteobacteria & Betaproteobacteria & Burkholderiales & Burkholderiaceae & Candidatus Glomeribacter \\
\hline Acidobacteria & Acidobacteriia & Acidobacteriales & Koribacteraceae & Candidatus Koribacter \\
\hline Chlamydiae & Chlamydiia & Chlamydiales & Parachlamydiaceae & Candidatus Protochlamydia \\
\hline Chlamydiae & Chlamydiia & Chlamydiales & Rhabdochlamydiaceae & Candidatus Rhabdochlamydia \\
\hline Acidobacteria & Solibacteres & Solibacterales & Solibacteraceae & Candidatus Solibacter \\
\hline Verrucomicrobia & [Spartobacteria] & [Chthoniobacterales] & [Chthoniobacteraceae] & Candidatus Xiphinematobacte \\
\hline Firmicutes & Bacilli & Lactobacillales & Carnobacteriaceae & Carnobacterium \\
\hline Actinobacteria & Actinobacteria & Actinomycetales & Micromonosporaceae & Catellatospora \\
\hline Actinobacteria & Actinobacteria & Actinomycetales & Micromonosporaceae & Catenuloplanes \\
\hline Proteobacteria & Alphaproteobacteria & Caulobacterales & Caulobacteraceae & Caulobacter \\
\hline Actinobacteria & Actinobacteria & Actinomycetales & Cellulomonadaceae & Cellulomonas \\
\hline Proteobacteria & Gammaproteobacteria & Alteromonadales & Alteromonadaceae & Cellvibrio \\
\hline Bacteroidetes & [Saprospirae] & [Saprospirales] & Chitinophagaceae & Chitinophaga \\
\hline Bacteroidetes & Flavobacteriia & Flavobacteriales & [Weeksellaceae] & Chryseobacterium \\
\hline Verrucomicrobia & [Spartobacteria] & [Chthoniobacterales] & [Chthoniobacteraceae] & Chthoniobacter \\
\hline Firmicutes & Clostridia & Clostridiales & Clostridiaceae & Clostridium \\
\hline Firmicutes & Bacilli & Bacillales & Paenibacillaceae & Cohnella \\
\hline Actinobacteria & Thermoleophilia & Solirubrobacterales & Conexibacteraceae & Conexibacter \\
\hline Firmicutes & Clostridia & Clostridiales & Lachnospiraceae & Coprococcus \\
\hline Proteobacteria & Gammaproteobacteria & Legionellales & Coxiellaceae & Coxiella \\
\hline Proteobacteria & Gammaproteobacteria & Methylococcales & Crenotrichaceae & Crenothrix \\
\hline Actinobacteria & Actinobacteria & Actinomycetales & Microbacteriaceae & Cryocola \\
\hline Proteobacteria & Betaproteobacteria & Burkholderiales & Oxalobacteraceae & Cupriavidus \\
\hline Bacteroidetes & Cytophagia & Cytophagales & Cytophagaceae & Cytophaga \\
\hline Verrucomicrobia & [Spartobacteria] & [Chthoniobacterales] & [Chthoniobacteraceae] & DA101 \\
\hline Actinobacteria & Actinobacteria & Actinomycetales & Micromonosporaceae & Dactylosporangium \\
\hline Proteobacteria & Betaproteobacteria & Rhodocyclales & Rhodocyclaceae & Dechloromonas \\
\hline Firmicutes & Clostridia & Clostridiales & Peptococcaceae & Dehalobacter_Syntrophobotul \\
\hline [Thermi] & Deinococci & Deinococcales & Deinococcaceae & Deinococcus \\
\hline Proteobacteria & Betaproteobacteria & Burkholderiales & Comamonadaceae & Delftia \\
\hline Actinobacteria & Actinobacteria & Actinomycetales & Dermacoccaceae & Dermacoccus \\
\hline Proteobacteria & Deltaproteobacteria & Desulfarculales & Desulfarculaceae & Desulfarculus \\
\hline
\end{tabular}




\begin{tabular}{|c|c|c|c|c|}
\hline 门 Phylum & 纲 Class & 目 Order & 科 Family & 属 Genus \\
\hline Proteobacteria & Deltaproteobacteria & Desulfobacterales & Desulfobulbaceae & Desulfobulbus \\
\hline Proteobacteria & Deltaproteobacteria & Syntrophobacterales & Syntrophaceae & Desulfomonile \\
\hline Firmicutes & Clostridia & Clostridiales & Peptococcaceae & Desulfosporosinus \\
\hline Proteobacteria & Deltaproteobacteria & Desulfovibrionales & Desulfovibrionaceae & Desulfovibrio \\
\hline Proteobacteria & Alphaproteobacteria & Rhizobiales & Hyphomicrobiaceae & Devosia \\
\hline Proteobacteria & Betaproteobacteria & Rhodocyclales & Rhodocyclaceae & Dok59 \\
\hline Proteobacteria & Gammaproteobacteria & Xanthomonadales & Xanthomonadaceae & Dokdonella \\
\hline Bacteroidetes & Cytophagia & Cytophagales & Cytophagaceae & Dyadobacter \\
\hline Bacteroidetes & Bacteroidia & Bacteroidales & Porphyromonadaceae & Dysgonomonas \\
\hline Acidobacteria & Acidobacteriia & Acidobacteriales & Acidobacteriaceae & Edaphobacter \\
\hline Verrucomicrobia & [Spartobacteria] & [Chthoniobacterales] & [Chthoniobacteraceae] & Ellin506 \\
\hline Proteobacteria & Gammaproteobacteria & Pseudomonadales & Moraxellaceae & Enhydrobacter \\
\hline Firmicutes & Clostridia & Clostridiales & Lachnospiraceae & Epulopiscium \\
\hline Proteobacteria & Gammaproteobacteria & Enterobacteriales & Enterobacteriaceae & Erwinia \\
\hline Chloroflexi & Ktedonobacteria & Ktedonobacterales & Ktedonobacteraceae & FFCH10602 \\
\hline Armatimonadetes & [Fimbriimonadia] & [Fimbriimonadales] & [Fimbriimonadaceae] & Fimbriimonas \\
\hline Bacteroidetes & [Saprospirae] & [Saprospirales] & Chitinophagaceae & Flavihumibacter \\
\hline Bacteroidetes & [Saprospirae] & [Saprospirales] & Chitinophagaceae & Flavisolibacter \\
\hline Bacteroidetes & Flavobacteriia & Flavobacteriales & Flavobacteriaceae & Flavobacterium \\
\hline Actinobacteria & Actinobacteria & Actinomycetales & Frankiaceae & Frankia \\
\hline Actinobacteria & Actinobacteria & Actinomycetales & Microbacteriaceae & Frigoribacterium \\
\hline Proteobacteria & Betaproteobacteria & Gallionellales & Gallionellaceae & Gallionella \\
\hline Planctomycetes & Planctomycetia & Gemmatales & Gemmataceae & Gemmata \\
\hline Gemmatimonadetes & Gemmatimonadetes & Gemmatimonadales & Gemmatimonadaceae & Gemmatimonas \\
\hline Firmicutes & Bacilli & Bacillales & Bacillaceae & Geobacillus \\
\hline Proteobacteria & Deltaproteobacteria & Desulfuromonadales & Geobacteraceae & Geobacter \\
\hline Actinobacteria & Actinobacteria & Actinomycetales & Geodermatophilaceae & Geodermatophilus \\
\hline Acidobacteria & Holophagae & Holophagales & Holophagaceae & Geothrix \\
\hline Nitrospirae & Nitrospira & Nitrospirales & [Thermodesulfovibrionaceae] & GOUTA19 \\
\hline Proteobacteria & Deltaproteobacteria & Myxococcales & Haliangiaceae & Haliangium \\
\hline Proteobacteria & Betaproteobacteria & Burkholderiales & Oxalobacteraceae & Herminiimonas \\
\hline Verrucomicrobia & [Spartobacteria] & [Chthoniobacterales] & [Chthoniobacteraceae] & heteroC45_4W \\
\hline Proteobacteria & Betaproteobacteria & Burkholderiales & Comamonadaceae & Hydrogenophaga \\
\hline Proteobacteria & Betaproteobacteria & Burkholderiales & Comamonadaceae & Hylemonella \\
\hline Bacteroidetes & Cytophagia & Cytophagales & Cytophagaceae & Hymenobacter \\
\hline Proteobacteria & Alphaproteobacteria & Rhizobiales & Hyphomicrobiaceae & Hyphomicrobium \\
\hline Actinobacteria & Acidimicrobiia & Acidimicrobiales & Iamiaceae & Iamia \\
\hline Proteobacteria & Alphaproteobacteria & Rhodospirillales & Rhodospirillaceae & Inquilinus \\
\hline Actinobacteria & Actinobacteria & Actinomycetales & Intrasporangiaceae & Janibacter \\
\hline Proteobacteria & Betaproteobacteria & Burkholderiales & Oxalobacteraceae & Janthinobacterium \\
\hline Nitrospirae & Nitrospira & Nitrospirales & Nitrospiraceae & JG37-AG-70 \\
\hline
\end{tabular}




\begin{tabular}{|c|c|c|c|c|}
\hline 门 Phylum & 纲 Class & 目 Order & 科 Family & 属 Genus \\
\hline Proteobacteria & Alphaproteobacteria & Rhizobiales & Rhizobiaceae & Kaistia \\
\hline Proteobacteria & Alphaproteobacteria & Sphingomonadales & Sphingomonadaceae & Kaistobacter \\
\hline Actinobacteria & Actinobacteria & Actinomycetales & Actinosynnemataceae & Kibdelosporangium \\
\hline Actinobacteria & Actinobacteria & Actinomycetales & Kineosporiaceae & Kineosporia \\
\hline Chloroflexi & Chloroflexi & [Roseiflexales] & [Kouleothrixaceae] & Kouleothrix \\
\hline Actinobacteria & Actinobacteria & Actinomycetales & Nocardioidaceae & Kribbella \\
\hline Actinobacteria & Actinobacteria & Actinomycetales & Actinosynnemataceae & Kutzneria \\
\hline Proteobacteria & Alphaproteobacteria & Rhizobiales & Xanthobacteraceae & Labrys \\
\hline Bacteroidetes & Cytophagia & Cytophagales & Cytophagaceae & Larkinella \\
\hline Proteobacteria & Gammaproteobacteria & Legionellales & Legionellaceae & Legionella \\
\hline Spirochaetes & [Leptospirae] & [Leptospirales] & Leptospiraceae & Leptonema \\
\hline Actinobacteria & Actinobacteria & Actinomycetales & Microbacteriaceae & Leucobacter \\
\hline Proteobacteria & Gammaproteobacteria & Xanthomonadales & Xanthomonadaceae & Luteibacter \\
\hline Proteobacteria & Gammaproteobacteria & Xanthomonadales & Xanthomonadaceae & Luteimonas \\
\hline Verrucomicrobia & Verrucomicrobiae & Verrucomicrobiales & Verrucomicrobiaceae & Luteolibacter \\
\hline Firmicutes & Bacilli & Bacillales & Planococcaceae & Lysinibacillus \\
\hline Proteobacteria & Gammaproteobacteria & Xanthomonadales & Xanthomonadaceae & Lysobacter \\
\hline Proteobacteria & Alphaproteobacteria & Rhodospirillales & Rhodospirillaceae & Magnetospirillum \\
\hline Proteobacteria & Gammaproteobacteria & Oceanospirillales & Oceanospirillaceae & Marinomonas \\
\hline Proteobacteria & Alphaproteobacteria & Rhizobiales & Phyllobacteriaceae & Mesorhizobium \\
\hline Proteobacteria & Betaproteobacteria & Burkholderiales & Comamonadaceae & Methylibium \\
\hline Proteobacteria & Alphaproteobacteria & Rhizobiales & Methylobacteriaceae & Methylobacterium \\
\hline Proteobacteria & Alphaproteobacteria & Rhizobiales & Beijerinckiaceae & Methyloferula \\
\hline Proteobacteria & Alphaproteobacteria & Rhizobiales & Methylocystaceae & Methylopila \\
\hline Proteobacteria & Alphaproteobacteria & Rhizobiales & Methylocystaceae & Methylosinus \\
\hline Proteobacteria & Betaproteobacteria & Methylophilales & Methylophilaceae & Methylotenera \\
\hline Actinobacteria & Actinobacteria & Actinomycetales & Microbacteriaceae & Microbacterium \\
\hline Actinobacteria & Actinobacteria & Actinomycetales & Geodermatophilaceae & Modestobacter \\
\hline Actinobacteria & Actinobacteria & Actinomycetales & Mycobacteriaceae & Mycobacterium \\
\hline Proteobacteria & Alphaproteobacteria & Caulobacterales & Caulobacteraceae & Mycoplana \\
\hline Proteobacteria & Deltaproteobacteria & Myxococcales & Myxococcaceae & Myхососсиs \\
\hline Proteobacteria & Deltaproteobacteria & Myxococcales & Nannocystaceae & Nannocystis \\
\hline Proteobacteria & Gammaproteobacteria & Xanthomonadales & Sinobacteraceae & Nevskia \\
\hline Bacteroidetes & [Saprospirae] & [Saprospirales] & Chitinophagaceae & Niastella \\
\hline Proteobacteria & Alphaproteobacteria & Rhizobiales & Bradyrhizobiaceae & Nitrobacter \\
\hline Proteobacteria & Betaproteobacteria & Nitrosomonadales & Nitrosomonadaceae & Nitrosovibrio \\
\hline Nitrospirae & Nitrospira & Nitrospirales & Nitrospiraceae & Nitrospira \\
\hline Actinobacteria & Actinobacteria & Actinomycetales & Nocardiaceae & Nocardia \\
\hline Actinobacteria & Actinobacteria & Actinomycetales & Nocardioidaceae & Nocardioides \\
\hline Actinobacteria & Actinobacteria & Actinomycetales & Streptosporangiaceae & Nonomuraea \\
\hline Planctomycetes & Planctomycetia & Gemmatales & Isosphaeraceae & Nostocoida \\
\hline
\end{tabular}




\begin{tabular}{|c|c|c|c|c|}
\hline 门 Phylum & 纲 Class & 目 Order & 科 Family & 属 Genus \\
\hline Proteobacteria & Alphaproteobacteria & Sphingomonadales & Sphingomonadaceae & Novosphingobium \\
\hline Proteobacteria & Alphaproteobacteria & Rhizobiales & Brucellaceae & Ochrobactrum \\
\hline Verrucomicrobia & Opitutae & Opitutales & Opitutaceae & Opitutus \\
\hline Verrucomicrobia & [Spartobacteria] & [Chthoniobacterales] & [Chthoniobacteraceae] & OR-59 \\
\hline Acidobacteria & Solibacteres & Solibacterales & Other & Other \\
\hline Acidobacteria & Acidobacteriia & Acidobacteriales & Acidobacteriaceae & Other \\
\hline Acidobacteria & Other & Other & Other & Other \\
\hline Acidobacteria & Acidobacteriia & Acidobacteriales & Koribacteraceae & Other \\
\hline Actinobacteria & Actinobacteria & Actinomycetales & Micromonosporaceae & Other \\
\hline Actinobacteria & Actinobacteria & Actinomycetales & Geodermatophilaceae & Other \\
\hline Actinobacteria & Actinobacteria & Actinomycetales & Other & Other \\
\hline Actinobacteria & Actinobacteria & Actinomycetales & Microbacteriaceae & Other \\
\hline Actinobacteria & Actinobacteria & Actinomycetales & Intrasporangiaceae & Other \\
\hline Actinobacteria & Thermoleophilia & Solirubrobacterales & Other & Other \\
\hline Actinobacteria & Actinobacteria & Actinomycetales & Actinosynnemataceae & Other \\
\hline Actinobacteria & Actinobacteria & Actinomycetales & Streptomycetaceae & Other \\
\hline Actinobacteria & Actinobacteria & Actinomycetales & Micrococcaceae & Other \\
\hline Actinobacteria & Actinobacteria & Actinomycetales & Streptosporangiaceae & Other \\
\hline Actinobacteria & Actinobacteria & Actinomycetales & Pseudonocardiaceae & Other \\
\hline Actinobacteria & Actinobacteria & Actinomycetales & Kineosporiaceae & Other \\
\hline Actinobacteria & Actinobacteria & Actinomycetales & Cryptosporangiaceae & Other \\
\hline Actinobacteria & Acidimicrobiia & Acidimicrobiales & Iamiaceae & Other \\
\hline Actinobacteria & Actinobacteria & Actinomycetales & Thermomonosporaceae & Other \\
\hline Actinobacteria & Actinobacteria & Actinomycetales & Cellulomonadaceae & Other \\
\hline Actinobacteria & Thermoleophilia & Gaiellales & Other & Other \\
\hline Actinobacteria & Acidimicrobiia & Acidimicrobiales & Other & Other \\
\hline Actinobacteria & Actinobacteria & Actinomycetales & Frankiaceae & Other \\
\hline AD3 & Other & Other & Other & Other \\
\hline Armatimonadetes & Chthonomonadetes & Chthonomonadales & Other & Other \\
\hline Armatimonadetes & Other & Other & Other & Other \\
\hline Armatimonadetes & Chthonomonadetes & Chthonomonadales & Chthonomonadaceae & Other \\
\hline Armatimonadetes & [Fimbriimonadia] & [Fimbriimonadales] & [Fimbriimonadaceae] & Other \\
\hline Bacteroidetes & [Saprospirae] & [Saprospirales] & Chitinophagaceae & Other \\
\hline Bacteroidetes & Sphingobacteriia & Sphingobacteriales & Other & Other \\
\hline Bacteroidetes & Other & Other & Other & Other \\
\hline Bacteroidetes & Cytophagia & Cytophagales & Cytophagaceae & Other \\
\hline Bacteroidetes & Sphingobacteriia & Sphingobacteriales & Sphingobacteriaceae & Other \\
\hline Bacteroidetes & Flavobacteriia & Flavobacteriales & Cryomorphaceae & Other \\
\hline Chlamydiae & Chlamydiia & Chlamydiales & Parachlamydiaceae & Other \\
\hline Chlamydiae & Chlamydiia & Chlamydiales & Other & Other \\
\hline Chlamydiae & Chlamydiia & Other & Other & Other \\
\hline
\end{tabular}




\begin{tabular}{|c|c|c|c|c|}
\hline 门 Phylum & 纲 Class & 目 Order & 科 Family & 属 Genus \\
\hline Chlamydiae & Chlamydiia & Chlamydiales & Waddliaceae & Other \\
\hline Chlamydiae & Chlamydiia & Chlamydiales & Simkaniaceae & Other \\
\hline Chloroflexi & Other & Other & Other & Other \\
\hline Chloroflexi & Anaerolineae & Other & Other & Other \\
\hline Chloroflexi & TK10 & B07_WMSP1 & Other & Other \\
\hline Chloroflexi & Anaerolineae & A31 & Other & Other \\
\hline Chloroflexi & Anaerolineae & Anaerolineales & Anaerolinaceae & Other \\
\hline Firmicutes & Bacilli & Bacillales & Planococcaceae & Other \\
\hline Firmicutes & Clostridia & Clostridiales & Peptostreptococcaceae & Other \\
\hline Firmicutes & Bacilli & Bacillales & Bacillaceae & Other \\
\hline Firmicutes & Bacilli & Bacillales & Thermoactinomycetaceae & Other \\
\hline Firmicutes & Clostridia & Clostridiales & Other & Other \\
\hline Firmicutes & Bacilli & Bacillales & Other & Other \\
\hline Firmicutes & Bacilli & Bacillales & Sporolactobacillaceae & Other \\
\hline Gemmatimonadetes & Gemmatimonadetes & Other & Other & Other \\
\hline Gemmatimonadetes & Gemmatimonadetes & Gemmatimonadales & Other & Other \\
\hline Nitrospirae & Nitrospira & Nitrospirales & Nitrospiraceae & Other \\
\hline Other & Other & Other & Other & Other \\
\hline Planctomycetes & Phycisphaerae & Phycisphaerales & Other & Other \\
\hline Proteobacteria & Betaproteobacteria & Burkholderiales & Oxalobacteraceae & Other \\
\hline Proteobacteria & Betaproteobacteria & Burkholderiales & Comamonadaceae & Other \\
\hline Proteobacteria & Betaproteobacteria & Other & Other & Other \\
\hline Proteobacteria & Alphaproteobacteria & Other & Other & Other \\
\hline Proteobacteria & Betaproteobacteria & Burkholderiales & Burkholderiaceae & Other \\
\hline Proteobacteria & Alphaproteobacteria & Rhizobiales & Hyphomicrobiaceae & Other \\
\hline Proteobacteria & Alphaproteobacteria & Rhizobiales & Rhizobiaceae & Other \\
\hline Proteobacteria & Alphaproteobacteria & Rhizobiales & Other & Other \\
\hline Proteobacteria & Alphaproteobacteria & Rhizobiales & Bradyrhizobiaceae & Other \\
\hline Proteobacteria & Gammaproteobacteria & Other & Other & Other \\
\hline Proteobacteria & Deltaproteobacteria & Myxococcales & Polyangiaceae & Other \\
\hline Proteobacteria & Betaproteobacteria & Rhodocyclales & Rhodocyclaceae & Other \\
\hline Proteobacteria & Deltaproteobacteria & Myxococcales & Other & Other \\
\hline Proteobacteria & Gammaproteobacteria & Legionellales & Other & Other \\
\hline Proteobacteria & Alphaproteobacteria & Rhizobiales & Beijerinckiaceae & Other \\
\hline Proteobacteria & Betaproteobacteria & Burkholderiales & Other & Other \\
\hline Proteobacteria & Gammaproteobacteria & Xanthomonadales & Sinobacteraceae & Other \\
\hline Proteobacteria & Gammaproteobacteria & Legionellales & Legionellaceae & Other \\
\hline Proteobacteria & Gammaproteobacteria & Xanthomonadales & Xanthomonadaceae & Other \\
\hline Proteobacteria & Alphaproteobacteria & Caulobacterales & Caulobacteraceae & Other \\
\hline Proteobacteria & Gammaproteobacteria & Enterobacteriales & Enterobacteriaceae & Other \\
\hline Proteobacteria & Alphaproteobacteria & Rhizobiales & Phyllobacteriaceae & Other \\
\hline
\end{tabular}




\begin{tabular}{|c|c|c|c|c|}
\hline 门 Phylum & 纲 Class & 目 Order & 科 Family & 属 Genus \\
\hline Proteobacteria & Alphaproteobacteria & Rickettsiales & mitochondria & Other \\
\hline Proteobacteria & Alphaproteobacteria & Rhizobiales & Methylocystaceae & Other \\
\hline Proteobacteria & Alphaproteobacteria & Rhodobacterales & Rhodobacteraceae & Other \\
\hline Proteobacteria & Other & Other & Other & Other \\
\hline Proteobacteria & Alphaproteobacteria & Rhizobiales & Brucellaceae & Other \\
\hline Proteobacteria & Betaproteobacteria & Methylophilales & Other & Other \\
\hline Proteobacteria & Deltaproteobacteria & Other & Other & Other \\
\hline Proteobacteria & Betaproteobacteria & Burkholderiales & Alcaligenaceae & Other \\
\hline Proteobacteria & Betaproteobacteria & Methylophilales & Methylophilaceae & Other \\
\hline Proteobacteria & Gammaproteobacteria & Alteromonadales & Alteromonadaceae & Other \\
\hline Proteobacteria & Alphaproteobacteria & Rhizobiales & Aurantimonadaceae & Other \\
\hline Proteobacteria & Alphaproteobacteria & Sphingomonadales & Sphingomonadaceae & Other \\
\hline Proteobacteria & Alphaproteobacteria & Rhodospirillales & Rhodospirillaceae & Other \\
\hline Proteobacteria & Alphaproteobacteria & Rhodospirillales & Acetobacteraceae & Other \\
\hline Proteobacteria & Alphaproteobacteria & Sphingomonadales & Other & Other \\
\hline Proteobacteria & Deltaproteobacteria & Desulfuromonadales & Other & Other \\
\hline Proteobacteria & Gammaproteobacteria & Chromatiales & Other & Other \\
\hline Proteobacteria & Gammaproteobacteria & Pseudomonadales & Moraxellaceae & Other \\
\hline Proteobacteria & Gammaproteobacteria & Pseudomonadales & Pseudomonadaceae & Other \\
\hline Proteobacteria & Betaproteobacteria & Neisseriales & Neisseriaceae & Other \\
\hline Proteobacteria & Gammaproteobacteria & Legionellales & Coxiellaceae & Other \\
\hline TM6 & SJA-4 & Other & Other & Other \\
\hline TM7 & Other & Other & Other & Other \\
\hline Verrucomicrobia & [Pedosphaerae] & [Pedosphaerales] & Other & Other \\
\hline Verrucomicrobia & Other & Other & Other & Other \\
\hline Verrucomicrobia & [Spartobacteria] & [Chthoniobacterales] & [Chthoniobacteraceae] & Other \\
\hline Verrucomicrobia & [Pedosphaerae] & [Pedosphaerales] & [Pedosphaeraceae] & Other \\
\hline WS3 & PRR-12 & Sediment-1 & Other & Other \\
\hline Proteobacteria & Betaproteobacteria & Burkholderiales & Oxalobacteraceae & Oxalobacter \\
\hline Firmicutes & Bacilli & Bacillales & Paenibacillaceae & Paenibacillus \\
\hline Firmicutes & Bacilli & Bacillales & Planococcaceae & Paenisporosarcina \\
\hline Proteobacteria & Betaproteobacteria & Burkholderiales & Burkholderiaceae & Pandoraea \\
\hline Chlamydiae & Chlamydiia & Chlamydiales & Parachlamydiaceae & Parachlamydia \\
\hline Proteobacteria & Alphaproteobacteria & Rhizobiales & Hyphomicrobiaceae & Parvibaculum \\
\hline Bacteroidetes & Sphingobacteriia & Sphingobacteriales & Sphingobacteriaceae & Pedobacter \\
\hline Proteobacteria & Alphaproteobacteria & Rhizobiales & Hyphomicrobiaceae & Pedomicrobium \\
\hline Verrucomicrobia & [Pedosphaerae] & [Pedosphaerales] & [Pedosphaeraceae] & Pedosphaera \\
\hline Proteobacteria & Deltaproteobacteria & Desulfuromonadales & Pelobacteraceae & Pelobacter \\
\hline Firmicutes & Clostridia & Clostridiales & Veillonellaceae & Pelosinus \\
\hline Proteobacteria & Gammaproteobacteria & Pseudomonadales & Moraxellaceae & Perlucidibaca \\
\hline Proteobacteria & Alphaproteobacteria & Rhodospirillales & Rhodospirillaceae & Phaeospirillum \\
\hline
\end{tabular}




\begin{tabular}{|c|c|c|c|c|}
\hline 门 Phylum & 纲 Class & 目 Order & 科 Family & 属 Genus \\
\hline Proteobacteria & Alphaproteobacteria & Caulobacterales & Caulobacteraceae & Phenylobacterium \\
\hline Actinobacteria & Actinobacteria & Actinomycetales & Intrasporangiaceae & Phycicoccus \\
\hline Proteobacteria & Alphaproteobacteria & Rhizobiales & Phyllobacteriaceae & Phyllobacterium \\
\hline Proteobacteria & Betaproteobacteria & Burkholderiales & Alcaligenaceae & Pigmentiphaga \\
\hline Actinobacteria & Actinobacteria & Actinomycetales & Micromonosporaceae & Pilimelia \\
\hline Planctomycetes & Planctomycetia & Pirellulales & Pirellulaceae & Pirellula \\
\hline Planctomycetes & Planctomycetia & Planctomycetales & Planctomycetaceae & Planctomyces \\
\hline Firmicutes & Bacilli & Bacillales & Thermoactinomycetaceae & Planifilum \\
\hline Proteobacteria & Alphaproteobacteria & Rhizobiales & Methylocystaceae & Pleomorphomonas \\
\hline Proteobacteria & Deltaproteobacteria & Myxococcales & Nannocystaceae & Plesiocystis \\
\hline Proteobacteria & Betaproteobacteria & Burkholderiales & Comamonadaceae & Polaromonas \\
\hline Proteobacteria & Betaproteobacteria & Procabacteriales & Procabacteriaceae & Procabacter \\
\hline Actinobacteria & Actinobacteria & Actinomycetales & Promicromonosporaceae & Promicromonospora \\
\hline Verrucomicrobia & Verrucomicrobiae & Verrucomicrobiales & Verrucomicrobiaceae & Prosthecobacter \\
\hline Firmicutes & Clostridia & Clostridiales & Clostridiaceae & Proteiniclasticum \\
\hline Proteobacteria & Gammaproteobacteria & Pseudomonadales & Pseudomonadaceae & Pseudomonas \\
\hline Actinobacteria & Actinobacteria & Actinomycetales & Pseudonocardiaceae & Pseudonocardia \\
\hline Proteobacteria & Gammaproteobacteria & Pseudomonadales & Moraxellaceae & Psychrobacter \\
\hline Bacteroidetes & Bacteroidia & Bacteroidales & Rikenellaceae & PW3 \\
\hline Proteobacteria & Betaproteobacteria & Burkholderiales & Comamonadaceae & Ramlibacter \\
\hline Actinobacteria & Actinobacteria & Actinomycetales & Microbacteriaceae & Rathayibacter \\
\hline Proteobacteria & Alphaproteobacteria & Rhizobiales & Rhizobiaceae & Rhizobium \\
\hline Proteobacteria & Gammaproteobacteria & Xanthomonadales & Xanthomonadaceae & Rhodanobacter \\
\hline Proteobacteria & Alphaproteobacteria & Rhodobacterales & Rhodobacteraceae & Rhodobacter \\
\hline Actinobacteria & Actinobacteria & Actinomycetales & Nocardiaceae & Rhodococcus \\
\hline Proteobacteria & Alphaproteobacteria & Rhizobiales & Hyphomicrobiaceae & Rhodoplanes \\
\hline Proteobacteria & Alphaproteobacteria & Rickettsiales & Rickettsiaceae & Rickettsia \\
\hline Proteobacteria & Gammaproteobacteria & Legionellales & Coxiellaceae & Rickettsiella \\
\hline Proteobacteria & Betaproteobacteria & Burkholderiales & Comamonadaceae & Roseateles \\
\hline Proteobacteria & Alphaproteobacteria & Rhodospirillales & Acetobacteraceae & Roseococcus \\
\hline Proteobacteria & Betaproteobacteria & Burkholderiales & Comamonadaceae & Rubrivivax \\
\hline Actinobacteria & Actinobacteria & Actinomycetales & Microbacteriaceae & Salinibacterium \\
\hline Proteobacteria & Betaproteobacteria & Burkholderiales & Burkholderiaceae & Salinispora \\
\hline Bacteroidetes & [Saprospirae] & [Saprospirales] & Chitinophagaceae & Sediminibacterium \\
\hline Bacteroidetes & [Saprospirae] & [Saprospirales] & Chitinophagaceae & Segetibacter \\
\hline Proteobacteria & Gammaproteobacteria & Enterobacteriales & Enterobacteriaceae & Serratia \\
\hline Firmicutes & Bacilli & Bacillales & Thermoactinomycetaceae & Shimazuella \\
\hline Proteobacteria & Alphaproteobacteria & Rhodospirillales & Rhodospirillaceae & Skermanella \\
\hline Firmicutes & Bacilli & Bacillales & Planococcaceae & Solibacillus \\
\hline Bacteroidetes & Sphingobacteriia & Sphingobacteriales & Sphingobacteriaceae & Solitalea \\
\hline Actinobacteria & Actinobacteria & Actinomycetales & Streptosporangiaceae & Sphaerisporangium \\
\hline
\end{tabular}




\begin{tabular}{|c|c|c|c|c|}
\hline 门 Phylum & 纲 Class & 目 Order & 科 Family & 属 Genus \\
\hline Bacteroidetes & Sphingobacteriia & Sphingobacteriales & Sphingobacteriaceae & Sphingobacterium \\
\hline Proteobacteria & Alphaproteobacteria & Sphingomonadales & Sphingomonadaceae & Sphingobium \\
\hline Proteobacteria & Alphaproteobacteria & Sphingomonadales & Sphingomonadaceae & Sphingomonas \\
\hline Proteobacteria & Alphaproteobacteria & Sphingomonadales & Sphingomonadaceae & Sphingopyxis \\
\hline Spirochaetes & Spirochaetes & Spirochaetales & Spirochaetaceae & Spirochaeta \\
\hline Bacteroidetes & Cytophagia & Cytophagales & Cytophagaceae & Spirosoma \\
\hline Actinobacteria & Actinobacteria & Actinomycetales & Sporichthyaceae & Sporichthya \\
\hline Bacteroidetes & Cytophagia & Cytophagales & Cytophagaceae & Sporocytophaga \\
\hline Firmicutes & Clostridia & Clostridiales & Veillonellaceae & Sporomusa \\
\hline Firmicutes & Bacilli & Bacillales & Planococcaceae & Sporosarcina \\
\hline Firmicutes & Bacilli & Bacillales & Staphylococcaceae & Staphylococcus \\
\hline Proteobacteria & Gammaproteobacteria & Xanthomonadales & Xanthomonadaceae & Stenotrophomonas \\
\hline Proteobacteria & Gammaproteobacteria & Xanthomonadales & Sinobacteraceae & Steroidobacter \\
\hline Proteobacteria & Betaproteobacteria & Rhodocyclales & Rhodocyclaceae & Sterolibacterium \\
\hline Actinobacteria & Actinobacteria & Actinomycetales & Streptomycetaceae & Streptacidiphilus \\
\hline Actinobacteria & Actinobacteria & Actinomycetales & Streptomycetaceae & Streptomyces \\
\hline Actinobacteria & Actinobacteria & Actinomycetales & Streptosporangiaceae & Streptosporangium \\
\hline Proteobacteria & Betaproteobacteria & Rhodocyclales & Rhodocyclaceae & Sulfuritalea \\
\hline Firmicutes & Clostridia & Clostridiales & Symbiobacteriaceae & Symbiobacterium \\
\hline Proteobacteria & Deltaproteobacteria & Syntrophobacterales & Syntrophobacteraceae & Syntrophobacter \\
\hline Proteobacteria & Gammaproteobacteria & Legionellales & Legionellaceae & Tatlockia \\
\hline Proteobacteria & Alphaproteobacteria & Rhodospirillales & Rhodospirillaceae & Telmatospirillum \\
\hline Firmicutes & Clostridia & Clostridiales & Peptostreptococcaceae & Tepidibacter \\
\hline Actinobacteria & Actinobacteria & Actinomycetales & Intrasporangiaceae & Terracoccus \\
\hline Acidobacteria & Acidobacteriia & Acidobacteriales & Acidobacteriaceae & Terriglobus \\
\hline Proteobacteria & Betaproteobacteria & Rhodocyclales & Rhodocyclaceae & Thauera \\
\hline Proteobacteria & Betaproteobacteria & Hydrogenophilales & Hydrogenophilaceae & Thiobacillus \\
\hline Firmicutes & Clostridia & Clostridiales & [Tissierellaceae] & Tissierella_Soehngenia \\
\hline Proteobacteria & Gammaproteobacteria & Enterobacteriales & Enterobacteriaceae & Trabulsiella \\
\hline Spirochaetes & Spirochaetes & Spirochaetales & Spirochaetaceae & Treponema \\
\hline Proteobacteria & Betaproteobacteria & Rhodocyclales & Rhodocyclaceae & TS34 \\
\hline Firmicutes & Bacilli & Turicibacterales & Turicibacteraceae & Turicibacter \\
\hline Spirochaetes & [Leptospirae] & [Leptospirales] & Leptospiraceae & Turneriella \\
\hline Proteobacteria & Betaproteobacteria & Rhodocyclales & Rhodocyclaceae & Uliginosibacterium \\
\hline Proteobacteria & Betaproteobacteria & Burkholderiales & Comamonadaceae & Variovorax \\
\hline Actinobacteria & Actinobacteria & Actinomycetales & Micromonosporaceae & Verrucosispora \\
\hline Actinobacteria & Actinobacteria & Actinomycetales & Micromonosporaceae & Virgisporangium \\
\hline Chlamydiae & Chlamydiia & Chlamydiales & Waddliaceae & Waddlia \\
\hline Proteobacteria & Alphaproteobacteria & Rickettsiales & Rickettsiaceae & Wolbachia \\
\hline Proteobacteria & Alphaproteobacteria & Rhizobiales & Xanthobacteraceae & Xanthobacter \\
\hline Proteobacteria & Betaproteobacteria & Rhodocyclales & Rhodocyclaceae & Zoogloea \\
\hline
\end{tabular}




\begin{tabular}{|c|c|c|c|c|}
\hline 门 Phylum & 纲 Class & 目 Order & 科 Family & 属 Genus \\
\hline- & - & - & - & - \\
\hline Acidobacteria & DA052 & Ellin6513 & - & - \\
\hline Acidobacteria & Acidobacteriia & Acidobacteriales & Koribacteraceae & - \\
\hline Acidobacteria & Solibacteres & Solibacterales & - & - \\
\hline Acidobacteria & [Chloracidobacteria] & RB41 & - & - \\
\hline Acidobacteria & iii1-8 & $32-20$ & - & - \\
\hline Acidobacteria & Acidobacteriia & Acidobacteriales & Acidobacteriaceae & - \\
\hline Acidobacteria & Acidobacteria-6 & iii1-15 & - & - \\
\hline Acidobacteria & iii1-8 & DS-18 & - & - \\
\hline Acidobacteria & Acidobacteria-6 & CCU21 & - & - \\
\hline Acidobacteria & Sva0725 & Sva0725 & - & - \\
\hline Acidobacteria & [Chloracidobacteria] & RB41 & Ellin6075 & - \\
\hline Acidobacteria & [Chloracidobacteria] & 42332 & - & - \\
\hline Acidobacteria & - & - & - & - \\
\hline Acidobacteria & S035 & - & - & - \\
\hline Acidobacteria & RB25 & - & - & - \\
\hline Acidobacteria & Acidobacteria-6 & iii1-15 & mb2424 & - \\
\hline Acidobacteria & TM1 & - & - & - \\
\hline Acidobacteria & Solibacteres & Solibacterales & Solibacteraceae & - \\
\hline Acidobacteria & Solibacteres & Solibacterales & PAUC26f & - \\
\hline Acidobacteria & iii1-8 & SJA-36 & - & - \\
\hline Acidobacteria & [Chloracidobacteria] & Ellin7246 & - & - \\
\hline Acidobacteria & [Chloracidobacteria] & DS-100 & - & - \\
\hline Acidobacteria & Acidobacteria-5 & - & - & - \\
\hline Acidobacteria & Solibacteres & JH-WHS99 & - & - \\
\hline Acidobacteria & BPC102 & MVS-40 & - & - \\
\hline Acidobacteria & Acidobacteria-6 & iii1-15 & RB40 & - \\
\hline Acidobacteria & AT-s54 & - & - & - \\
\hline Acidobacteria & [Chloracidobacteria] & PK29 & - & - \\
\hline Acidobacteria & EC1113 & - & - & - \\
\hline Acidobacteria & GAL08 & - & - & - \\
\hline Acidobacteria & Holophagae & Holophagales & Holophagaceae & - \\
\hline Acidobacteria & Solibacteres & Solibacterales & [Bryobacteraceae] & - \\
\hline Acidobacteria & PAUC37f & - & - & - \\
\hline Acidobacteria & Solibacteres & Solibacterales & AKIW659 & - \\
\hline Acidobacteria & BPC102 & - & - & - \\
\hline Acidobacteria & [Chloracidobacteria] & - & - & - \\
\hline Acidobacteria & iii1-8 & - & - & - \\
\hline Acidobacteria & Acidobacteriia & Acidobacteriales & - & - \\
\hline Actinobacteria & Thermoleophilia & Gaiellales & Gaiellaceae & - \\
\hline Actinobacteria & Thermoleophilia & Solirubrobacterales & - & - \\
\hline
\end{tabular}




\begin{tabular}{|c|c|c|c|c|}
\hline 门 Phylum & 纲 Class & 目 Order & 科 Family & 属 Genus \\
\hline Actinobacteria & Acidimicrobiia & Acidimicrobiales & EB1017 & - \\
\hline Actinobacteria & Actinobacteria & Actinomycetales & - & - \\
\hline Actinobacteria & Acidimicrobiia & Acidimicrobiales & - & - \\
\hline Actinobacteria & Actinobacteria & Actinomycetales & Micrococcaceae & - \\
\hline Actinobacteria & MB-A2-108 & 0319-7L14 & - & - \\
\hline Actinobacteria & Acidimicrobiia & Acidimicrobiales & C111 & - \\
\hline Actinobacteria & Thermoleophilia & Gaiellales & - & - \\
\hline Actinobacteria & Actinobacteria & Actinomycetales & Frankiaceae & - \\
\hline Actinobacteria & Actinobacteria & Actinomycetales & Micromonosporaceae & - \\
\hline Actinobacteria & Thermoleophilia & Solirubrobacterales & Conexibacteraceae & - \\
\hline Actinobacteria & Thermoleophilia & Solirubrobacterales & Solirubrobacteraceae & - \\
\hline Actinobacteria & Actinobacteria & Actinomycetales & Nocardioidaceae & - \\
\hline Actinobacteria & Thermoleophilia & Solirubrobacterales & Patulibacteraceae & - \\
\hline Actinobacteria & Actinobacteria & Actinomycetales & Pseudonocardiaceae & - \\
\hline Actinobacteria & Actinobacteria & Actinomycetales & Nakamurellaceae & - \\
\hline Actinobacteria & MB-A2-108 & - & - & - \\
\hline Actinobacteria & Actinobacteria & Actinomycetales & Microbacteriaceae & - \\
\hline Actinobacteria & Actinobacteria & Actinomycetales & Streptomycetaceae & - \\
\hline Actinobacteria & Actinobacteria & Actinomycetales & Sporichthyaceae & - \\
\hline Actinobacteria & Actinobacteria & Actinomycetales & Actinospicaceae & - \\
\hline Actinobacteria & Rubrobacteria & Rubrobacterales & Rubrobacteraceae & - \\
\hline Actinobacteria & Actinobacteria & Actinomycetales & Kineosporiaceae & - \\
\hline Actinobacteria & Actinobacteria & Actinomycetales & Intrasporangiaceae & - \\
\hline Actinobacteria & Actinobacteria & Micrococcales & - & - \\
\hline Actinobacteria & Actinobacteria & Actinomycetales & Thermomonosporaceae & - \\
\hline Actinobacteria & Thermoleophilia & Gaiellales & AK1AB1_02E & - \\
\hline Actinobacteria & Actinobacteria & Actinomycetales & Cryptosporangiaceae & - \\
\hline Actinobacteria & Acidimicrobiia & Acidimicrobiales & AKIW874 & - \\
\hline Actinobacteria & Acidimicrobiia & Acidimicrobiales & Microthrixaceae & - \\
\hline Actinobacteria & Actinobacteria & Actinomycetales & Actinosynnemataceae & - \\
\hline Actinobacteria & Acidimicrobiia & Acidimicrobiales & JdFBGBact & - \\
\hline Actinobacteria & Actinobacteria & Actinomycetales & Geodermatophilaceae & - \\
\hline Actinobacteria & OPB41 & - & - & - \\
\hline Actinobacteria & Actinobacteria & Actinomycetales & ACK-M1 & - \\
\hline AD3 & ABS-6 & - & - & - \\
\hline AD3 & JG37-AG-4 & - & - & - \\
\hline AD3 & - & - & - & - \\
\hline Armatimonadetes & Chthonomonadetes & Chthonomonadales & Chthonomonadaceae & - \\
\hline Armatimonadetes & 0319-6E2 & - & - & - \\
\hline Armatimonadetes & [Fimbriimonadia] & [Fimbriimonadales] & [Fimbriimonadaceae] & - \\
\hline Armatimonadetes & Armatimonadia & FW68 & - & - \\
\hline
\end{tabular}




\begin{tabular}{|c|c|c|c|c|}
\hline 门 Phylum & 纲 Class & 目 Order & 科 Family & 属 Genus \\
\hline Armatimonadetes & Chthonomonadetes & SJA-22 & - & - \\
\hline Armatimonadetes & Armatimonadia & Armatimonadales & Armatimonadaceae & - \\
\hline Armatimonadetes & OPB50 & - & - & - \\
\hline Armatimonadetes & [Fimbriimonadia] & [Fimbriimonadales] & - & - \\
\hline Armatimonadetes & SHA-37 & - & - & - \\
\hline Armatimonadetes & SJA-176 & RB046 & - & - \\
\hline Armatimonadetes & SJA-176 & - & - & - \\
\hline Armatimonadetes & SJA-176 & GAB-B06 & - & - \\
\hline Bacteroidetes & [Saprospirae] & [Saprospirales] & Chitinophagaceae & - \\
\hline Bacteroidetes & Sphingobacteriia & Sphingobacteriales & - & - \\
\hline Bacteroidetes & Sphingobacteriia & Sphingobacteriales & Sphingobacteriaceae & - \\
\hline Bacteroidetes & Cytophagia & Cytophagales & Cytophagaceae & - \\
\hline Bacteroidetes & [Saprospirae] & [Saprospirales] & - & - \\
\hline Bacteroidetes & [Saprospirae] & [Saprospirales] & Saprospiraceae & - \\
\hline Bacteroidetes & At12OctB3 & - & - & - \\
\hline Bacteroidetes & Flavobacteriia & Flavobacteriales & Cryomorphaceae & - \\
\hline Bacteroidetes & Bacteroidia & Bacteroidales & - & - \\
\hline Bacteroidetes & Bacteroidia & Bacteroidales & Rikenellaceae & - \\
\hline Bacteroidetes & Cytophagia & Cytophagales & [Amoebophilaceae] & - \\
\hline Bacteroidetes & VC2_1_Bac22 & - & - & - \\
\hline Bacteroidetes & Bacteroidia & Bacteroidales & GZKB119 & - \\
\hline Bacteroidetes & Cytophagia & Cytophagales & - & - \\
\hline BHI80-139 & - & - & - & - \\
\hline BRC1 & PRR-11 & - & - & - \\
\hline BRC1 & NPL-UPA2 & - & - & - \\
\hline Chlamydiae & Chlamydiia & Chlamydiales & - & - \\
\hline Chlamydiae & Chlamydiia & Chlamydiales & Parachlamydiaceae & - \\
\hline Chlamydiae & Chlamydiia & - & - & - \\
\hline Chlamydiae & Chlamydiia & Chlamydiales & Waddliaceae & - \\
\hline Chlamydiae & Chlamydiia & Chlamydiales & Chlamydiaceae & - \\
\hline Chlorobi & - & - & - & - \\
\hline Chlorobi & SJA-28 & - & - & - \\
\hline Chlorobi & OPB56 & - & - & - \\
\hline Chlorobi & BSV26 & A89 & - & - \\
\hline Chlorobi & BSV26 & PK329 & - & - \\
\hline Chlorobi & BSV26 & $\mathrm{C} 20$ & - & - \\
\hline Chlorobi & Ignavibacteria & Ignavibacteriales & Ignavibacteriaceae & - \\
\hline Chloroflexi & Ellin6529 & - & - & - \\
\hline Chloroflexi & TK10 & B07_WMSP1 & FFCH4570 & - \\
\hline Chloroflexi & Anaerolineae & SBR1031 & oc28 & - \\
\hline Chloroflexi & S085 & - & - & - \\
\hline
\end{tabular}




\begin{tabular}{|c|c|c|c|c|}
\hline 门 Phylum & 纲 Class & 目 Order & 科 Family & 属 Genus \\
\hline Chloroflexi & Ktedonobacteria & Thermogemmatisporales & Thermogemmatisporaceae & - \\
\hline Chloroflexi & Anaerolineae & SBR1031 & $\mathrm{A} 4 \mathrm{~b}$ & - \\
\hline Chloroflexi & Anaerolineae & H39 & - & - \\
\hline Chloroflexi & Chloroflexi & [Roseiflexales] & [Kouleothrixaceae] & - \\
\hline Chloroflexi & TK17 & - & - & - \\
\hline Chloroflexi & Ktedonobacteria & JG30-KF-AS9 & - & - \\
\hline Chloroflexi & TK10 & B07_WMSP1 & - & - \\
\hline Chloroflexi & TK10 & AKYG885 & $5 B-12$ & - \\
\hline Chloroflexi & C0119 & - & - & - \\
\hline Chloroflexi & Anaerolineae & A31 & S47 & - \\
\hline Chloroflexi & Gitt-GS-136 & - & - & - \\
\hline Chloroflexi & Anaerolineae & CFB-26 & - & - \\
\hline Chloroflexi & TK17 & mle1-48 & - & - \\
\hline Chloroflexi & Ktedonobacteria & Ktedonobacterales & Ktedonobacteraceae & - \\
\hline Chloroflexi & TK10 & AKYG885 & Dolo_23 & - \\
\hline Chloroflexi & Ktedonobacteria & TK10 & - & - \\
\hline Chloroflexi & Anaerolineae & SHA-20 & - & - \\
\hline Chloroflexi & Anaerolineae & envOPS12 & - & - \\
\hline Chloroflexi & Thermomicrobia & JG30-KF-CM45 & - & - \\
\hline Chloroflexi & Anaerolineae & WCHB1-50 & - & - \\
\hline Chloroflexi & TK10 & - & - & - \\
\hline Chloroflexi & Anaerolineae & SB-34 & - & - \\
\hline Chloroflexi & Chloroflexi & [Roseiflexales] & - & - \\
\hline Chloroflexi & Anaerolineae & Caldilineales & Caldilineaceae & - \\
\hline Chloroflexi & Thermomicrobia & Ellin6537 & - & - \\
\hline Chloroflexi & Anaerolineae & S0208 & - & - \\
\hline Chloroflexi & Ktedonobacteria & - & - & - \\
\hline Chloroflexi & Anaerolineae & GCA004 & - & - \\
\hline Chloroflexi & Ktedonobacteria & B12-WMSP1 & - & - \\
\hline Chloroflexi & SHA-26 & - & - & - \\
\hline Chloroflexi & Chloroflexi & AKIW781 & - & - \\
\hline Chloroflexi & SAR202 & - & - & - \\
\hline Chloroflexi & P2-11E & - & - & - \\
\hline Chloroflexi & Anaerolineae & - & - & - \\
\hline Chloroflexi & - & - & - & - \\
\hline Chloroflexi & Thermomicrobia & AKYG1722 & - & - \\
\hline Chloroflexi & Anaerolineae & A31 & - & - \\
\hline Chloroflexi & Anaerolineae & SBR1031 & SJA-101 & - \\
\hline Chloroflexi & Anaerolineae & pLW-97 & - & - \\
\hline Chloroflexi & Chloroflexi & Chloroflexales & 01D2Z48 & - \\
\hline Chloroflexi & Chloroflexi & Chloroflexales & FFCH7168 & - \\
\hline
\end{tabular}




\begin{tabular}{|c|c|c|c|c|}
\hline 门 Phylum & 纲 Class & 目 Order & 科 Family & 属 Genus \\
\hline Chloroflexi & Ktedonobacteria & Elev-1554 & - & - \\
\hline Chloroflexi & Anaerolineae & Anaerolineales & Anaerolinaceae & - \\
\hline Chloroflexi & Thermomicrobia & - & - & - \\
\hline Chloroflexi & Anaerolineae & SBR1031 & SHA-31 & - \\
\hline Chloroflexi & Chloroflexi & Herpetosiphonales & - & - \\
\hline Cyanobacteria & 4C0d-2 & MLE1-12 & - & - \\
\hline Cyanobacteria & ML635J-21 & - & - & - \\
\hline Cyanobacteria & Chloroplast & Stramenopiles & - & - \\
\hline Cyanobacteria & 4C0d-2 & SM1D11 & - & - \\
\hline Cyanobacteria & Chloroplast & Streptophyta & - & - \\
\hline Cyanobacteria & Chloroplast & Chlorophyta & - & - \\
\hline Elusimicrobia & Elusimicrobia & FAC88 & - & - \\
\hline Elusimicrobia & Elusimicrobia & IIb & - & - \\
\hline Elusimicrobia & Elusimicrobia & Elusimicrobiales & - & - \\
\hline Elusimicrobia & Endomicrobia & - & - & - \\
\hline Elusimicrobia & Elusimicrobia & MVP-88 & - & - \\
\hline Elusimicrobia & - & - & - & - \\
\hline FBP & - & - & - & - \\
\hline FCPU426 & - & - & - & - \\
\hline Fibrobacteres & Fibrobacteria & 258ds10 & - & - \\
\hline Fibrobacteres & SBZC_2415 & - & - & - \\
\hline Firmicutes & Bacilli & Bacillales & Bacillaceae & - \\
\hline Firmicutes & Bacilli & Bacillales & Planococcaceae & - \\
\hline Firmicutes & Clostridia & Clostridiales & Ruminococcaceae & - \\
\hline Firmicutes & Bacilli & Bacillales & - & - \\
\hline Firmicutes & Clostridia & Clostridiales & Lachnospiraceae & - \\
\hline Firmicutes & Clostridia & Clostridiales & Peptostreptococcaceae & - \\
\hline Firmicutes & Clostridia & Clostridiales & Clostridiaceae & - \\
\hline Firmicutes & Bacilli & Bacillales & Thermoactinomycetaceae & - \\
\hline Firmicutes & Clostridia & Clostridiales & - & - \\
\hline Firmicutes & Clostridia & Clostridiales & Gracilibacteraceae & - \\
\hline Firmicutes & Bacilli & Bacillales & Alicyclobacillaceae & - \\
\hline Firmicutes & Clostridia & OPB54 & - & - \\
\hline Firmicutes & Bacilli & Bacillales & [Exiguobacteraceae] & - \\
\hline Firmicutes & Clostridia & Clostridiales & Veillonellaceae & - \\
\hline Firmicutes & Bacilli & Bacillales & Paenibacillaceae & - \\
\hline Firmicutes & Clostridia & Clostridiales & Christensenellaceae & - \\
\hline GAL15 & - & - & - & - \\
\hline Gemmatimonadetes & Gemm-1 & - & - & - \\
\hline Gemmatimonadetes & Gemmatimonadetes & N1423WL & - & - \\
\hline Gemmatimonadetes & Gemmatimonadetes & Ellin5290 & - & - \\
\hline
\end{tabular}


赵爱花，杜晓军，㶓婧，张守仁，焦志华。宝天曼落叶阔叶林土壤细菌多样性. 生物多样性，2015，23 (5)：649-657. http://www. biodiversity-science. net/CN/article/downloadArticleFile. do?attachType=PDF\&id=10069

\begin{tabular}{|c|c|c|c|c|}
\hline 门 Phylum & 纲 Class & 目 Order & 科 Family & 属 Genus \\
\hline Gemmatimonadetes & Gemmatimonadetes & - & - & - \\
\hline Gemmatimonadetes & Gemmatimonadetes & Gemmatimonadales & Ellin5301 & - \\
\hline Gemmatimonadetes & Gemmatimonadetes & KD8-87 & - & - \\
\hline Gemmatimonadetes & Gemm-5 & - & - & - \\
\hline Gemmatimonadetes & Gemmatimonadetes & C114 & - & - \\
\hline Gemmatimonadetes & Gemm-2 & - & - & - \\
\hline Gemmatimonadetes & Gemmatimonadetes & Gemmatimonadales & - & - \\
\hline Gemmatimonadetes & Gemm-3 & - & - & - \\
\hline Gemmatimonadetes & - & - & - & - \\
\hline GN02 & GN07 & - & - & - \\
\hline GN02 & $3 B R-5 F$ & - & - & - \\
\hline GN02 & GKS2-174 & - & - & - \\
\hline GN02 & BB34 & - & - & - \\
\hline GN04 & MSB-5A5 & - & - & - \\
\hline GN04 & - & - & - & - \\
\hline GOUTA4 & - & - & - & - \\
\hline Kazan-3B-28 & - & - & - & - \\
\hline MVP-21 & - & - & - & - \\
\hline Nitrospirae & Nitrospira & Nitrospirales & 0319-6A21 & - \\
\hline Nitrospirae & Nitrospira & Nitrospirales & [Leptospirillaceae] & - \\
\hline Nitrospirae & Nitrospira & Nitrospirales & Nitrospiraceae & - \\
\hline Nitrospirae & Nitrospira & Nitrospirales & - & - \\
\hline Nitrospirae & Nitrospira & Nitrospirales & $4-29$ & - \\
\hline NKB19 & - & - & - & - \\
\hline NKB19 & TSBW08 & - & - & - \\
\hline OD1 & ZB2 & - & - & - \\
\hline OD1 & SM2F11 & - & - & - \\
\hline OD1 & - & - & - & - \\
\hline OD1 & ABY1 & - & - & - \\
\hline OD1 & Mb-NB09 & - & - & - \\
\hline OP11 & OP11-3 & - & - & - \\
\hline OP11 & - & - & - & - \\
\hline OP11 & WCHB1-64 & d153 & - & - \\
\hline OP11 & OP11-4 & - & - & - \\
\hline OP11 & WCHB1-64 & - & - & - \\
\hline OP11 & OP11-1 & - & - & - \\
\hline OP3 & koll11 & - & - & - \\
\hline OP3 & PBS-25 & - & - & - \\
\hline OP3 & koll11 & GIF10 & kpj58rc & - \\
\hline PAUC34f & - & - & - & - \\
\hline Planctomycetes & Planctomycetia & Gemmatales & Gemmataceae & - \\
\hline
\end{tabular}




\begin{tabular}{|c|c|c|c|c|}
\hline 门 Phylum & 纲 Class & 目 Order & 科 Family & 属 Genus \\
\hline Planctomycetes & Phycisphaerae & WD2101 & - & - \\
\hline Planctomycetes & Planctomycetia & Gemmatales & Isosphaeraceae & - \\
\hline Planctomycetes & Planctomycetia & Pirellulales & Pirellulaceae & - \\
\hline Planctomycetes & OM190 & agg27 & - & - \\
\hline Planctomycetes & Phycisphaerae & Phycisphaerales & - & - \\
\hline Planctomycetes & BD7-11 & - & - & - \\
\hline Planctomycetes & Phycisphaerae & CPla-3 & - & - \\
\hline Planctomycetes & vadinHA49 & p04_C01 & - & - \\
\hline Planctomycetes & Phycisphaerae & S-70 & - & - \\
\hline Planctomycetes & Phycisphaerae & Pla1 & - & - \\
\hline Planctomycetes & - & - & - & - \\
\hline Planctomycetes & C6 & MVS-107 & - & - \\
\hline Planctomycetes & OM190 & CL500-15 & - & - \\
\hline Planctomycetes & Phycisphaerae & mle1-8 & - & - \\
\hline Planctomycetes & Pla3 & - & - & - \\
\hline Planctomycetes & Pla4 & - & - & - \\
\hline Planctomycetes & 028H05-P-BN-P5 & - & - & - \\
\hline Planctomycetes & Phycisphaerae & CCM11a & - & - \\
\hline Planctomycetes & vadinHA49 & DH61 & - & - \\
\hline Planctomycetes & Phycisphaerae & Phycisphaerales & Phycisphaeraceae & - \\
\hline Planctomycetes & Planctomycetia & B97 & - & - \\
\hline Planctomycetes & C6 & d113 & - & - \\
\hline Planctomycetes & OM190 & - & - & - \\
\hline Planctomycetes & Phycisphaerae & AKAU3564 & - & - \\
\hline Planctomycetes & ODP123 & - & - & - \\
\hline Planctomycetes & Phycisphaerae & C86 & - & - \\
\hline Proteobacteria & Gammaproteobacteria & Xanthomonadales & Sinobacteraceae & - \\
\hline Proteobacteria & Alphaproteobacteria & Rhodospirillales & Rhodospirillaceae & - \\
\hline Proteobacteria & Alphaproteobacteria & Rhizobiales & Bradyrhizobiaceae & - \\
\hline Proteobacteria & Deltaproteobacteria & Syntrophobacterales & Syntrophobacteraceae & - \\
\hline Proteobacteria & Betaproteobacteria & - & - & - \\
\hline Proteobacteria & Alphaproteobacteria & Ellin329 & - & - \\
\hline Proteobacteria & Gammaproteobacteria & Enterobacteriales & Enterobacteriaceae & - \\
\hline Proteobacteria & Deltaproteobacteria & Myxococcales & - & - \\
\hline Proteobacteria & Betaproteobacteria & A21b & EB1003 & - \\
\hline Proteobacteria & Alphaproteobacteria & Rhizobiales & Hyphomicrobiaceae & - \\
\hline Proteobacteria & Betaproteobacteria & Ellin6067 & - & - \\
\hline Proteobacteria & Betaproteobacteria & SC-I-84 & - & - \\
\hline Proteobacteria & Gammaproteobacteria & Xanthomonadales & Xanthomonadaceae & - \\
\hline Proteobacteria & Alphaproteobacteria & Rhizobiales & - & - \\
\hline Proteobacteria & Alphaproteobacteria & - & - & - \\
\hline
\end{tabular}




\begin{tabular}{|c|c|c|c|c|}
\hline 门 Phylum & 纲 Class & 目 Order & 科 Family & 属 Genus \\
\hline Proteobacteria & Alphaproteobacteria & Rhodospirillales & Acetobacteraceae & - \\
\hline Proteobacteria & Betaproteobacteria & Burkholderiales & Comamonadaceae & - \\
\hline Proteobacteria & Deltaproteobacteria & Myxococcales & Haliangiaceae & - \\
\hline Proteobacteria & Betaproteobacteria & MND1 & - & - \\
\hline Proteobacteria & Betaproteobacteria & Burkholderiales & Oxalobacteraceae & - \\
\hline Proteobacteria & Gammaproteobacteria & Legionellales & Coxiellaceae & - \\
\hline Proteobacteria & Alphaproteobacteria & Rhizobiales & Methylocystaceae & - \\
\hline Proteobacteria & Betaproteobacteria & IS-44 & - & - \\
\hline Proteobacteria & Alphaproteobacteria & Caulobacterales & Caulobacteraceae & - \\
\hline Proteobacteria & Deltaproteobacteria & Myxococcales & Myхососсасеае & - \\
\hline Proteobacteria & Alphaproteobacteria & Rhodobacterales & Hyphomonadaceae & - \\
\hline Proteobacteria & Alphaproteobacteria & Rhizobiales & Beijerinckiaceae & - \\
\hline Proteobacteria & Betaproteobacteria & Burkholderiales & Alcaligenaceae & - \\
\hline Proteobacteria & Gammaproteobacteria & Thiotrichales & Piscirickettsiaceae & - \\
\hline Proteobacteria & Gammaproteobacteria & Legionellales & - & - \\
\hline Proteobacteria & Deltaproteobacteria & MIZ46 & - & - \\
\hline Proteobacteria & Alphaproteobacteria & Sphingomonadales & Sphingomonadaceae & - \\
\hline Proteobacteria & Deltaproteobacteria & Myxococcales & Polyangiaceae & - \\
\hline Proteobacteria & Deltaproteobacteria & - & - & - \\
\hline Proteobacteria & Gammaproteobacteria & HTCC2188 & HTCC2089 & - \\
\hline Proteobacteria & Deltaproteobacteria & NB1-j & NB1-i & - \\
\hline Proteobacteria & Deltaproteobacteria & NB1-j & MND4 & - \\
\hline Proteobacteria & Alphaproteobacteria & BD7-3 & - & - \\
\hline Proteobacteria & Deltaproteobacteria & [Entotheonellales] & [Entotheonellaceae] & - \\
\hline Proteobacteria & Alphaproteobacteria & Rhizobiales & Phyllobacteriaceae & - \\
\hline Proteobacteria & Alphaproteobacteria & Rickettsiales & - & - \\
\hline Proteobacteria & Gammaproteobacteria & Chromatiales & Ectothiorhodospiraceae & - \\
\hline Proteobacteria & Deltaproteobacteria & NB1-j & - & - \\
\hline Proteobacteria & Deltaproteobacteria & Spirobacillales & - & - \\
\hline Proteobacteria & Betaproteobacteria & A21b & UD5 & - \\
\hline Proteobacteria & Betaproteobacteria & Burkholderiales & - & - \\
\hline Proteobacteria & Alphaproteobacteria & Rhizobiales & Xanthobacteraceae & - \\
\hline Proteobacteria & Deltaproteobacteria & Myxococcales & OM27 & - \\
\hline Proteobacteria & Betaproteobacteria & Nitrosomonadales & Nitrosomonadaceae & - \\
\hline Proteobacteria & TA18 & CV90 & - & - \\
\hline Proteobacteria & Deltaproteobacteria & FAC87 & - & - \\
\hline Proteobacteria & Betaproteobacteria & Methylophilales & - & - \\
\hline Proteobacteria & Alphaproteobacteria & Rhizobiales & Rhizobiaceae & - \\
\hline Proteobacteria & Betaproteobacteria & Rhodocyclales & Rhodocyclaceae & - \\
\hline Proteobacteria & Alphaproteobacteria & Rhodospirillales & - & - \\
\hline Proteobacteria & Betaproteobacteria & Methylophilales & Methylophilaceae & - \\
\hline
\end{tabular}




\begin{tabular}{|c|c|c|c|c|}
\hline 门 Phylum & 纲 Class & 目 Order & 科 Family & 属 Genus \\
\hline Proteobacteria & TA18 & PHOS-HD29 & - & - \\
\hline Proteobacteria & Gammaproteobacteria & - & - & - \\
\hline Proteobacteria & Deltaproteobacteria & Myxococcales & Cystobacterineae & - \\
\hline Proteobacteria & Gammaproteobacteria & Legionellales & Legionellaceae & - \\
\hline Proteobacteria & Alphaproteobacteria & Sphingomonadales & Erythrobacteraceae & - \\
\hline Proteobacteria & Gammaproteobacteria & Chromatiales & - & - \\
\hline Proteobacteria & - & - & - & - \\
\hline Proteobacteria & Alphaproteobacteria & Rickettsiales & Rickettsiaceae & - \\
\hline Proteobacteria & Betaproteobacteria & Procabacteriales & Procabacteriaceae & - \\
\hline Proteobacteria & Deltaproteobacteria & Myxococcales & 0319-6G20 & - \\
\hline Proteobacteria & Betaproteobacteria & Burkholderiales & Burkholderiaceae & - \\
\hline Proteobacteria & Betaproteobacteria & SBla14 & - & - \\
\hline Proteobacteria & Deltaproteobacteria & Bdellovibrionales & Bdellovibrionaceae & - \\
\hline Proteobacteria & Betaproteobacteria & Nitrosomonadales & - & - \\
\hline Proteobacteria & Gammaproteobacteria & Alteromonadales & OM60 & - \\
\hline Proteobacteria & Betaproteobacteria & Neisseriales & Neisseriaceae & - \\
\hline Proteobacteria & Deltaproteobacteria & Syntrophobacterales & Syntrophaceae & - \\
\hline Proteobacteria & Gammaproteobacteria & Acidithiobacillales & - & - \\
\hline Proteobacteria & Deltaproteobacteria & Bdellovibrionales & Bacteriovoracaceae & - \\
\hline Proteobacteria & Alphaproteobacteria & Sphingomonadales & - & - \\
\hline Proteobacteria & Betaproteobacteria & Thiobacterales & - & - \\
\hline Proteobacteria & Deltaproteobacteria & Sva0853 & JTB36 & - \\
\hline Proteobacteria & Deltaproteobacteria & NB1-j & JTB38 & - \\
\hline Proteobacteria & Alphaproteobacteria & Rhodobacterales & Rhodobacteraceae & - \\
\hline Proteobacteria & Gammaproteobacteria & Pseudomonadales & Moraxellaceae & - \\
\hline Proteobacteria & Gammaproteobacteria & Alteromonadales & 125ds10 & - \\
\hline Proteobacteria & Deltaproteobacteria & Desulfobacterales & Desulfobulbaceae & - \\
\hline Proteobacteria & Deltaproteobacteria & Desulfovibrionales & Desulfovibrionaceae & - \\
\hline Proteobacteria & Deltaproteobacteria & AF420338 & - & - \\
\hline Proteobacteria & Deltaproteobacteria & PB19 & - & - \\
\hline Proteobacteria & Gammaproteobacteria & PYR10d3 & - & - \\
\hline Proteobacteria & Alphaproteobacteria & Kiloniellales & - & - \\
\hline Proteobacteria & Alphaproteobacteria & Rhizobiales & Methylobacteriaceae & - \\
\hline Proteobacteria & Gammaproteobacteria & Aeromonadales & Aeromonadaceae & - \\
\hline Proteobacteria & Gammaproteobacteria & Alteromonadales & 211ds20 & - \\
\hline Proteobacteria & Gammaproteobacteria & Pseudomonadales & Pseudomonadaceae & - \\
\hline Proteobacteria & Gammaproteobacteria & Thiotrichales & - & - \\
\hline Proteobacteria & Gammaproteobacteria & [Marinicellales] & [Marinicellaceae] & - \\
\hline Proteobacteria & Alphaproteobacteria & Rhizobiales & Aurantimonadaceae & - \\
\hline Proteobacteria & Alphaproteobacteria & Rhizobiales & Bartonellaceae & - \\
\hline Proteobacteria & Alphaproteobacteria & Rickettsiales & mitochondria & - \\
\hline
\end{tabular}




\begin{tabular}{|c|c|c|c|c|}
\hline 门 Phylum & 纲 Class & 目 Order & 科 Family & 属 Genus \\
\hline Proteobacteria & Deltaproteobacteria & GMD14H09 & - & - \\
\hline Proteobacteria & Deltaproteobacteria & NKB15 & - & - \\
\hline Proteobacteria & Zetaproteobacteria & Mariprofundales & Mariprofundaceae & - \\
\hline SBR1093 & - & - & - & - \\
\hline Spirochaetes & Spirochaetes & Spirochaetales & Spirochaetaceae & - \\
\hline Spirochaetes & [Brevinematae] & [Brevinematales] & - & - \\
\hline Spirochaetes & GN05 & - & - & - \\
\hline Tenericutes & Mollicutes & - & - & - \\
\hline TM6 & SJA-4 & - & - & - \\
\hline TM6 & SBRH58 & - & - & - \\
\hline TM6 & SJA-4 & S1198 & - & - \\
\hline TM7 & TM7-1 & - & - & - \\
\hline TM7 & SC3 & - & - & - \\
\hline TM7 & TM7-3 & - & - & - \\
\hline TM7 & TM7-3 & EW055 & - & - \\
\hline TM7 & - & - & - & - \\
\hline TM7 & MJK10 & - & - & - \\
\hline TM7 & TM7-3 & I025 & - & - \\
\hline TM7 & TM7-3 & Blgi18 & - & - \\
\hline Verrucomicrobia & [Pedosphaerae] & [Pedosphaerales] & Ellin515 & - \\
\hline Verrucomicrobia & [Pedosphaerae] & [Pedosphaerales] & - & - \\
\hline Verrucomicrobia & [Pedosphaerae] & [Pedosphaerales] & auto67_4W & - \\
\hline Verrucomicrobia & [Methylacidiphilae] & S-BQ2-57 & - & - \\
\hline Verrucomicrobia & [Pedosphaerae] & [Pedosphaerales] & Ellin517 & - \\
\hline Verrucomicrobia & [Spartobacteria] & [Chthoniobacterales] & [Chthoniobacteraceae] & - \\
\hline Verrucomicrobia & [Methylacidiphilae] & Methylacidiphilales & - & - \\
\hline Verrucomicrobia & Verrucomicrobiae & Verrucomicrobiales & Verrucomicrobiaceae & - \\
\hline Verrucomicrobia & Opitutae & Opitutales & Opitutaceae & - \\
\hline Verrucomicrobia & [Pedosphaerae] & [Pedosphaerales] & R4-41B & - \\
\hline Verrucomicrobia & [Pedosphaerae] & [Pedosphaerales] & OPB35 & - \\
\hline Verrucomicrobia & [Pedosphaerae] & [Pedosphaerales] & [Pedosphaeraceae] & - \\
\hline Verrucomicrobia & - & - & - & - \\
\hline Verrucomicrobia & Verruco-5 & LD1-PB3 & - & - \\
\hline Verrucomicrobia & Opitutae & [Cerasicoccales] & [Cerasicoccaceae] & - \\
\hline Verrucomicrobia & Verruco-5 & WCHB1-41 & - & - \\
\hline Verrucomicrobia & Opitutae & - & - & - \\
\hline Verrucomicrobia & [Methylacidiphilae] & Methylacidiphilales & LD19 & - \\
\hline Verrucomicrobia & [Spartobacteria] & [Chthoniobacterales] & 01D2Z36 & - \\
\hline WPS-2 & - & - & - & - \\
\hline WS2 & SHA-109 & - & - & - \\
\hline WS2 & Kazan-3B-09 & - & - & - \\
\hline
\end{tabular}


赵爱花，杜晓军，藏婧，张守仁，焦志华. 宝天曼落叶阔叶林土壤细菌多样性. 生物多样性，2015，23 (5)：649-657.

http://www. biodiversity-science. net/CN/article/downloadArticleFile. do?attachType=PDF\&id=10069

\begin{tabular}{lllll}
\hline 门 Phylum & 纲 Class & 目 Order & 科 Family & 属 Genus \\
\hline WS3 & PRR-12 & Sediment-1 & PRR-10 & - \\
WS3 & PRR-12 & Sediment-1 & - & - \\
WS3 & PRR-12 & LD1-PA13 & - & - \\
WS3 & PRR-12 & - & - & - \\
WS3 & PRR-12 & Sediment-1 & CV106 & - \\
WS3 & PRR-12 & wb1_H11 & - & - \\
WS4 & - & - & - & - \\
ZB3 & BS119 & - & - & - \\
\hline
\end{tabular}


附录2 宝天曼森林动态监测样地 31 个土壤样品中检测到的不同分类水平上细菌优势类群及其相对丰度(其 中Unknown代表未鉴定出的类群, Others代表丰度低于1\%的类群)

Appendix 2 Relative abundances of the dominant bacteria at different taxonomic levels in the 31 soil samples in the Baotianman Forest Dynamics Plot. Unknown means the taxa that can not be identified; Others represent the taxa whose relative abundances are less than $1 \%$.
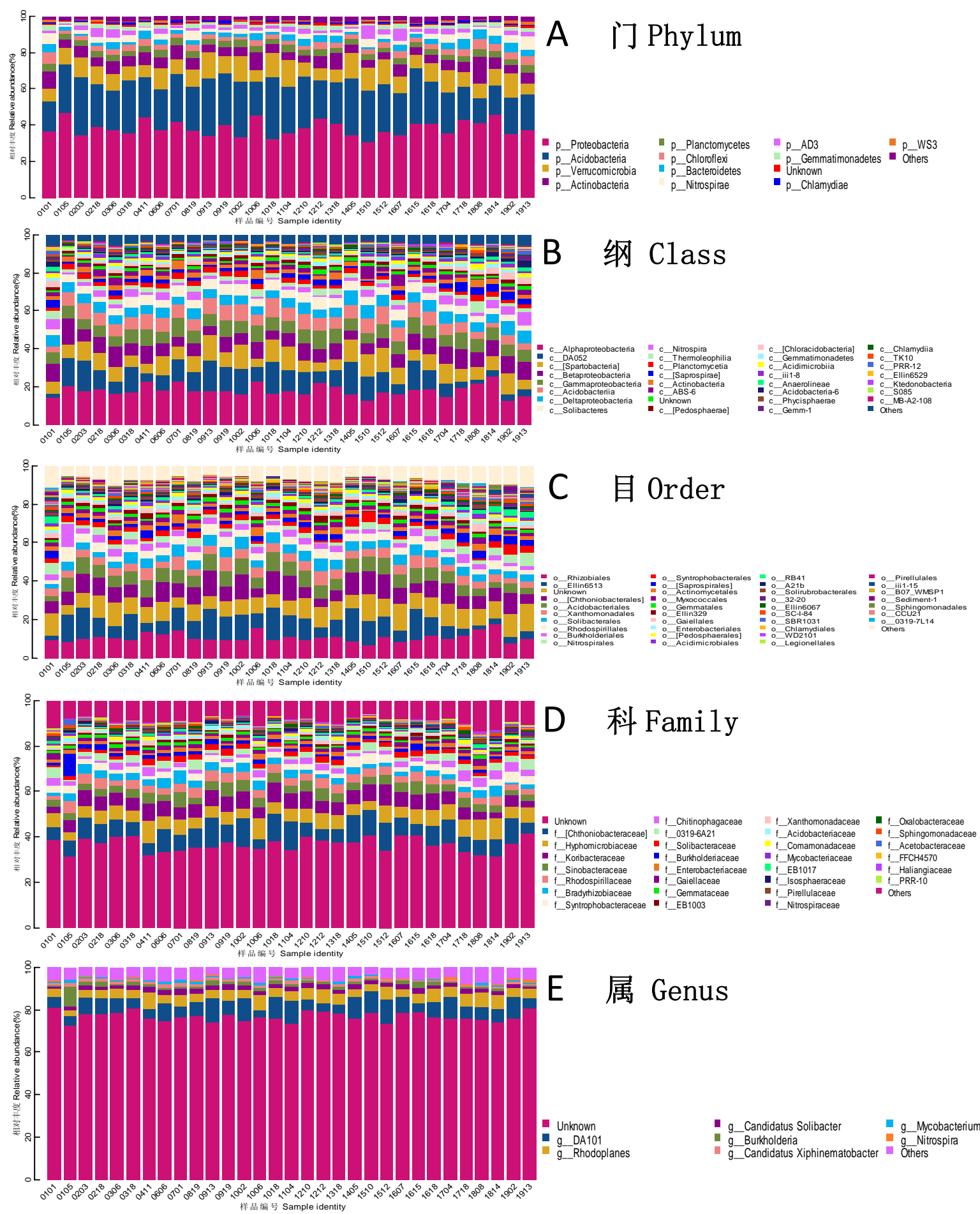

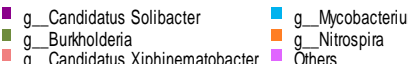

